\title{
Formes de piété individuelles et collectives dans le royaume du Bosphore cimmérien. L'exemple des inscriptions votives
}

\section{Ivonne Ohlerich}

Traducteur : Laurent Vallance

\section{OpenEdition}

Journals

Édition électronique

URL : https://journals.openedition.org/edl/1727

DOI : $10.4000 /$ edl. 1727

ISSN : 2296-5084

\section{Éditeur}

Université de Lausanne

\section{Édition imprimée}

Date de publication : 15 mai 2019

Pagination : 243-284

ISBN : 978-2-940331-70-3

ISSN : 0014-2026

\section{Référence électronique}

Ivonne Ohlerich, «Formes de piété individuelles et collectives dans le royaume du Bosphore cimmérien. L'exemple des inscriptions votives », Études de lettres [En ligne], 309 | 2019, mis en ligne le 15 mai 2021, consulté le 17 mai 2021. URL : http://journals.openedition.org/edl/1727 ; DOI : https:// doi.org/10.4000/edl.1727 


\section{FORMES DE PIÉTÉ INDIVIDUELLES ET COLLECTIVES DANS LE ROYAUME DU BOSPHORE CIMMÉRIEN. L'EXEMPLE DES INSCRIPTIONS VOTIVES}

La piété est un acte religieux et fonde la cohésion sociale. Aussi une marque de piété publique peut-elle s'expliquer par beaucoup de raisons, qu'il s'agisse de montrer son appartenance à un groupe social ou de présenter une image de soi. La tradition grecque antique connaissait de nombreux moyens d'exprimer sa piété. La plus individuelle est sans doute l'inscription votive, exposée dans l'espace public. Dans le futur royaume du Bosphore Cimmérien, habité par des colons grecs au VIe s. av. J.-C., des inscriptions votives étaient exposées depuis le $\mathrm{IV}^{\mathrm{e}}$ siècle. On les considère en général comme un témoignage de la floraison économique du royaume. Mais est-ce seulement faute de moyens financiers que les colons n'ont pas introduit plus tôt la tradition grecque? Un examen des inscriptions votives et de l'évolution de la religion et des sanctuaires dans le royaume du Bosphore prouve que l'on choisissait très consciemment les moyens d'exprimer sa foi et de se présenter soi-même, conférant ainsi à cette culture un caractère tout à fait particulier.

\section{Introduction}

Le royaume du Bosphore Cimmérien s'étendait sur toute la péninsule de Kertch - la pointe orientale de la Crimée - et sur la péninsule de Taman, située en face et qui dans l'Antiquité était un archipel ${ }^{1}$. Au nord, le royaume du Bosphore confinait à la mer d'Azov, au sud, à la mer Noire. Après la fondation des premières colonies et sous-colonies sur la côte nord-est de la mer Noire au cours du VIe s. av. J.-C., le royaume a évolué

I. Dernière mise à jour concernant la géographie de la région dans l'Antiquité: U. Schlotzhauer, D. V. Žuravlev, "Forschungen in der antiken Siedlung „, Golubickaja-2 “ auf der südrussischen Taman'-Halbinsel». 
durant le $\mathrm{V}^{\mathrm{e}}$ s. av. J.-C. pour devenir un État régi par une monarchie, dont le siège se trouvait à Panticapée ${ }^{2}$.

La première phase de colonisation est pour l'essentiel encore obscure. La métropole des nouveaux colonisateurs ne peut être déterminée avec certitude que dans de rares cas ${ }^{3}$. On ne sait donc pas quelle croyance et quels rituels les colons ont amenés avec eux de leur patrie. Les colonies, à l'origine indépendantes les unes des autres, entretenaient leurs propres sanctuaires et exploitaient les terres des territoires qui en dépendaient.

Selon Diodore, c'est vers 480 que la dynastie des Archéanactides a étendu son pouvoir sur une partie des colonies du Pont septentrional en mettant pied dans la partie asiatique du futur royaume, la péninsule de Taman ${ }^{4}$. La dynastie des Spartocides arrive au pouvoir deux décennies plus tard seulement et préside durant plus de deux siècles aux destinées du royaume. C'est alors que celui-ci atteint son extension maximale. L'union des colonies, y compris leur territoire environnant et les nombreuses souscolonies qu'elles avaient fondées entretemps ${ }^{5}$, parfois réalisée sous une forme violente ${ }^{6}$, contribua à des conditions économiques et politiques plus stables. En même temps que les nouvelles structures sociales s'établissent, l'aspiration des habitants du royaume du Bosphore augmente à exprimer leur piété de manière visible et représentative. Cela est particulièrement

2. Diod. XII.31.1; Strab. VII.4.4; V. D. Blavatskij, Pantikapej, p. 7 ; J. G. Vinogradov, "Die historische Entwicklung der Poleis des nördlichen Schwarzmeergebietes im 5. Jahrhundert v. Chr.», p. 67 sq.; V. P. Tolstikov, «K probleme obrazovanija Bosporskogo gosudarstva», en part. p. 43 sq.

3. C'est Milet qui passe pour être la métropole des implantations de Panticapée, Kèpoi et Théodosie sur la péninsule de Kertch: Strab. VII.4.4; Ps.-Skym. 899; Plin. HNVI.18; Arr. Peripl. M. Eux. 19.3; Ps. Arr. Peripl. M. Eux. 77.

4. Diod. XII.31.1.

5. Sur le statut des implantations du Bosphore: V. F. Gajdukevič, Das Bosporanische Reich, p. 22 sq.; Th. S. Noonan, "The origins of the Greek colony at Pantikapaeum»; D. B. Šelov, «Istorija», p. 9 sq.; G. A. Košelenko, V. D. Kuznecov, "Grečeskaja kolonizacija Bospora» (1985 et 1992) et "Greek colonisation of the Bosporus», p. 252.

6. Théodosie à la limite occidentale de la péninsule de Kertch: D. B. Šelov, «K voprosu o vzaimodejstvii grečeskikh i mestnykh kul'tov v Severnom Pričernomor'e»; V. F. Gajdukevič, Das Bosporanische Reich, p. 70 sq.; Nymphaion au sud de Panticapée: Aeschin, Ctes. 171f.; L. F. Silant'eva, "Nekropol' Nimfeja», p. 96; M. M. Khudjak, Iz istorii Nimfeja VI-III vekov do n. è., p. 31; D. B. Šelov, "Istorija», p. 13; V. A. Anokhin, Monetnoe delo Bospora, p. 19; I. E. Surikov, «Historico-geographical questions connected with Pericle's pontic expedition», p. 361; V. N. Zin'ko, «Khora Nimfeja v VI-IV vv. do n. è.", p. 89. 
manifeste dans les inscriptions votives qui nous sont conservées. Elles proviennent de la région du royaume du Bosphore et datent du $\mathrm{IV}^{\mathrm{e}} \mathrm{s}$. av. J.-C. ${ }^{7}$, soit plus de deux siècles après la fondation des premières colonies ${ }^{8}$.

\section{Chronologie et diffusion des inscriptions votives}

Il faut d'abord commencer par examiner de plus près ces inscriptions du Bosphore. Elles suivent un schéma homogène: en général, sont indiqués la divinité honorée et le dédicant, souvent avec son patronyme. Les inscriptions plus détaillées précisent en outre le nom du monarque régnant et les peuples sujets à son autorité. Le motif de l'offrande et le nom de la personne qu'elle entend honorer sont parfois mentionnés également.

Nous avons à peu près autant d'inscriptions provenant de l'une et de l'autre péninsule - de Kertch et de Taman (fig. 1). La capitale du royaume occupe une place particulière: à une seule exception, toutes les inscriptions votives de la péninsule de Kertch proviennent de Panticapée. Pour ce qui est de la péninsule de Taman, il $\mathrm{y}$ a au moins quatre centres où des divinités étaient honorées par des inscriptions - les cités de Hermonassa, Phanagorie, Gorgippia et le sanctuaire extra-urbain sur la montagne de «Boris et Gleb» (outre le sanctuaire d'Aphrodite Ourania à Apatouron, seulement attesté par des sources écrites et non localisé à ce jour).

Dans les deux parties du royaume, on observe la même évolution chronologique: sur l'une et l'autre péninsule, les premières inscriptions conservées remontent à la fin du $\mathrm{V}^{\mathrm{e}}$ s. av. J.-C. Suit un âge d'or de la tradition, que reflète le nombre relativement élevé d'inscriptions conservées. Dans une phase d'essor économique, où les contacts politiques et économiques avec Athènes sont étroits ${ }^{9}$, les inscriptions votives connaissent pour un

7. Les inscriptions funéraires sont en usage dès le $\mathrm{V}^{\mathrm{e}}$ s. av. J.-C. Voir p. ex. CIRB $152,153$.

8. V. F. Gajdukevič, Das Bosporanische Reich, p. 15 sq.; K. K. Marčenko, "Osnovnye aspekty i rezul'taty izučenija greko-varvarskikh kontaktov i vzaimodejstvij v Severnom Pričernomor'e skifskoj èpokhi ", p. 334. Les fouilles les plus récentes sur l'acropole de Panticapée témoignent en faveur d'une implantation des premiers colons grecs dès la fin du VII s. av. J.-C. : N. S. Astašova et al., «Drevnejšij Pantikapej - Predvaritel'nye itogi arkheologičeskikh issledovanij».

9. A. V. Podossinov, "Am Rande der griechischen Oikumene. Geschichte des Bosporanischen Reiches», p. 28-31. 
246

ÉTUDES DE LETTRES

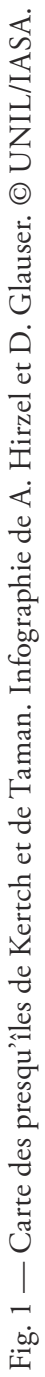


bon siècle un grand succès. Après le maximum atteint au IV ${ }^{\mathrm{e}} \mathrm{s}$. av. J.-C. avec 28 inscriptions sur un total de 48 , leur nombre recule sensiblement au III ${ }^{\mathrm{e}}$ s. av. J.-C. (12 inscriptions conservées seulement). Seules quatre inscriptions sont datées du $\mathrm{II}^{\mathrm{e}}$ s. av. J.-C.

Les deux péninsules ont un autre point commun: le culte à Apollon Iètros ${ }^{10} \mathrm{y}$ était à peu près autant pratiqué. Considéré comme le mélange d'un culte thérapeutique thrace et de traditions grecques ${ }^{11}$, ce culte, probablement importé de la mer Noire occidentale, est attesté dans presque tous les établissements bosporans pour lesquels on dispose de sources épigraphiques ${ }^{12}$. Une prêtrise pour la divinité est en outre bien attestée $^{13}$. À Panticapée, la famille régnante des Spartocides a fourni ellemême plusieurs prêtres, ce qui prouve la grande importance politique de ce culte à l'échelle du royaume, un culte attesté jusqu'à la fin de la dynastie, dans les dernières années du II e s. av. J.-C.

Dans l'une et l'autre partie du royaume, Aphrodite était la divinité dont le culte était le plus populaire. À Panticapée, dans les premières inscriptions la mentionnant, elle apparaît sans épiclèse ${ }^{14}$. Dans quatre des cinq inscriptions de la péninsule de Taman ${ }^{15}$, on l'honore

IO. Panticapée: CIRB Al'bom 6, 10, 25; V. F. Gajdukevič, Das Bosporanische Reich, p. 90 sq. Sous son règne, de 240 à 220 av. J.-C., Leukôn II a fait frapper à Panticapée des monnaies de différentes dénominations à l'effigie d'Apollon: cf. V. A. Anokhin, Monetnoe delo Bospora, p. 48 sq., nos 133-147. Hermonassa: CIRB Al'bom 1037, 1038. N. S. Belova ("Novaja nadpis' iz Germonassy») a confirmé que les noms de famille des dédicants sont purement grecs et considère pour cette raison qu'il s'agit d'un culte authentiquement grec; Phanagorie: CIRB Al'bom 974.

II. Pour la thèse que le culte de Iètros depuis Histria, son foyer originel sur la mer Noire, se serait diffusé vers l'orient et aurait rencontré un tel succès au nord du PontEuxin grâce à sa compatibilité avec les courants religieux thraces et locaux: Y. Ustinova, "Apollo Iatros». Sur ce culte en général: V. F. Gajdukevič, Das Bosporanische Reich, p. 177; A. S. Rusjaeva, "Milet-Didimy-Borisfen-Ol'vija», p. 33 sq.; N. Erhardt, "Apollon Ietros»; A. S. Rusjaeva, "Feb Apollon na Bospore»; M. V. Skržinskaja, "Bosporjane na prazdnikakh Apollona».

I2. Panticapée: CIRB Al'bom 6, 10, 25, Ch. Löhr, Griechische Familienweihungen, cat. no 97; Hermonassa: CIRB Al'bom 1037, 1044; Phanagorie: CIRB Al'bom 974.

I3. Outre la prêtrise d'un Spartoklès - nom qui renvoie à la famille régnante -, fils de Dynostratès (CIRB Al'bom 6), plus tard, au III ${ }^{\mathrm{e}}$ s. av. J.-C., on a l'exemple du roi Leukôn II, fils de Pairisadès II, qui a consacré une statue au dieu à l'occasion de son accession à la prêtrise: CIRB 25.

I4. CIRB Al'bom 7, 13, 17, 75.

I5. N. I. Sokol'skij, "Svjatilišče Afrodity v Kepakh», p. 116; N. S. Belova, «Posvjatitel'naja nadpis' iz Kep». 
de l'épiclèse "Ourania qui règne sur Apatouron " ${ }^{16}$, dont on n'a pas d'exemple en dehors du royaume du Bosphore. Bien que provenant de différents sites, elles se réfèrent au fameux sanctuaire d'Aphrodite Ourania, non encore localisé, qui se trouvait sur la partie asiatique du royaume du Bosphore ${ }^{17}$ - d'après Strabon sur le territoire voisin des Sindes ${ }^{18}$. Si en Grèce, l'épiclèse Ourania est attestée pour différentes divinités, Apatouron était probablement le nom du lieu-dit où se trouvait le sanctuaire ${ }^{19}$, et donc une épiclèse limitée d'abord à la partie orientale du royaume. On peut considérer le culte syncrétique de la divinité honorée à Apatouron comme le résultat du contact interculturel entre les colons grecs et la population indigène. Sur la foi de nombreuses représentations retrouvées uniquement dans l'enceinte des kourganes et caractérisées par des conceptions religieuses ${ }^{20}$ fortement étrangères à la culture grecque $^{21}$, la déesse elle-même est identifiée avec une divinité tantôt

I6. Phanagorie: CIRB Al'bom 971 Apollodoros, fils de Phanagoros a consacré à Aphrodite Ourania Apatouron Medeousa. Dans la consécration d'une statue par une femme nommée Kassalia, fille de Posios (CIRB Al'bom 972), la déesse porte juste l'épithète Ourania: B. von Köhne, «Über die Marmorbasis aus der Zeit des bosporanischen Königs Pairisades I»; péninsule de Taman: l'inscription CIRB Al'bom 1111 qui figure sur un piédestal a été retrouvée avec deux fragments de statues au pied de la salse «Boris et Gleb». CIRB Al'bom 1234 - lieu de découverte inconnu - est la plus ancienne consécration connue à cette divinité.

17. L. P. Kharko, "Kul't Afrodity na Bospore Kimmerijskom»; N. P. Rozanova, «K voprosu o mestonakhoždenii Apatur»; I. D. Marčenko, "K voprosu o kul'takh Aziatskogo Bospora» et «Novye dannye o dogrečeskom Pantikapee»; V. F. Gajdukevič, Das Bosporanische Reich, p. 225 sq.; I. D. Marčenko, "O kul'te Afrodity na Tamane»; S. R. Tokhtas'ev, "Bosporskaja legenda ob Afrodite Apaturos» et "Apatur»; Y. Ustinova, The supreme gods of the bosporan kingdom, p. 32 sq.; K. Stähler, "Die antike Siedlung Vyšesteblievskaja 1", p. 119 sq.

I8. Strab. IV.2.10.

I9. N. P. Rozanova, "K voprosu o mestonakhoždenii Apatur», p. 212 sq.; V. F. Gajdukevič, Das Bosporanische Reich, p. 228; K. Stähler, "Die antike Siedlung Vyšesteblievskaja 1", p. 119 sq. Sur cette discussion en général: S. R. Tokhtas'ev, "Apatur», p. 141.

20. A. P. Iwanowa, "Gestalten der örtlichen Mythologie in der Kunst des Bosporos»; I. D. Marčenko, «K voprosu o kul'takh Aziatskogo Bospora»; I. J. Šaub, «Kul't velikoj bogini u mestnogo naselenija Severnogo Pričernomor' ja»; I. A. Emec, Greko-Varvarskie religioznye vzaimovlijanija na Bospore Kimmerijskom, p. 154-156; N. V. Moleva, Očerki sakral'noj žizni Bospora, p. 79-84.

2I. A. P. Iwanowa, "Gestalten der örtlichen Mythologie in der Kunst des Bosporos", p. 235 sq.; S. S. Bessonova, «Kul't Tabiti-Gestii u Skifov». 
aux jambes en forme de serpent tantôt assise en majesté sur un trône. À Panticapée ${ }^{22}$, cette épiclèse n'apparaît qu'au II ${ }^{\mathrm{e}}$ s. av. J.-C., Aphrodite n'étant pas attestée dans les inscriptions votives du III s. av. J.-C.

Sur la péninsule de Kertch comme sur celle de Taman, par ailleurs, les mêmes divinités paraissent avoir été honorées à parts à peu près égales, à en juger par les inscriptions à Héraclès ${ }^{23}$, Déméter ${ }^{24}$ et Dionysos ${ }^{25}$. Seule Artémis semble avoir été plus populaire à Kertch, avec quatre inscriptions conservées ${ }^{26}$ qui attestent son culte, contre deux sur la péninsule de Taman, une à Gorgippia ${ }^{27}$ et une à Hermonassa ${ }^{28}$.

22. CIRB Al'bom 75. La plus ancienne consécration connue d'un thiase à Panticapée a été trouvée sur le flanc nord-est du mont Mithridate. Elle figure sur une dalle calcaire décorée de bas-reliefs, dont la face supérieure forme un dièdre où Aphrodite est représentée chevauchant un cygne ou une oie: Y. Ustinova, The supreme gods of the bosporan kingdom, p. 46 sq. et table 4. L'inscription atteste la consécration par Théoritès, fils de Démétrios, et par d'autres thiasotes à Aphrodite Ourania, maîtresse d'Apatouron, pour l'archonte et roi Pairisadès, fils de Pairisadès, la reine Kamasarye, la fille de Spartokos et son époux Agrotès, fils d'Isanthès.

23. Panticapée: CIRB Al'bom 16; Phanagorie: CIRB Al'bom 973.

24. On suppose que deux des trois consécrations de Panticapée conservées sont liées à une prêtrisee. L'inscription CIRB Al'bom 8 sur une plaque de marbre avec une consécration par Kreusa à Déméter n’est que partiellement conservée. Pour la deuxième consécration (CIRB Al'bom 14), faite à Déméter pour sa fille Démétrie par la prêtresse Aristoniké, la prêtrise est certaine mais non l'origine: Ch. Löhr, Griechische Familienweihungen, p. 106 sq., cat. no 126; troisième inscription: CIRB Al'bom 18.

25. Panticapée: CIRB Al'bom 15, 24, une inscription monumentale de Nymphaion par laquelle l'agonothète Megaclès consacre une porte à Dionysos. Cette inscription a été découverte à proximité immédiate du portique d'un lieu de culte qui n’a pas encore été fouillé: O. J. Sokolova, N. A. Pavličenko, «Novaja posvjatitel'naja nadpis' iz Nimfeja», p. 101 ; O. J. Sokolova, "Lučšie nakhodki iz raskopok Nimfeja za poslednie desjat' let", p. 93.

26. Deux des consécrations sont le fait d'une femme: la fille d'une certaine Nymphaia a offert un autel à Artémis après avoir quitté sa charge de prêtresse (CIRB Al'bom 6a). Korana, fille d'Itia, a consacré une statue de sujet inconnu à Artémis d'Éphèse (CIRB Al'bom 11). Dans deux autres inscriptions, Artémis est honorée sans épiclèse spécifique: un certain Phénippe lui consacre une statue en l'honneur de son frère (CIRB Al'bom 9 - Ch. Löhr, Griechische Familienweihungen, cat. no 144), un dédicant inconnu honore la divinité également par une statue (CIRB Al'bom 12).

27. CIRB Al'bom 1114.

28. Le fragment d'un pied de fauteuil en marbre nous a transmis la consécration d'un prêtre ou d'une prêtresse d'Hermonassa à Artémis, datant du règne de Pairisadès Ier (CIRB Al'bom 1040). 
Les inscriptions représentent une large palette sociale. Évidemment, ce sont surtout les élites locales qui s'y mettent en avant. À Panticapée, nous l'avons dit, plusieurs membres de la maison royale ont occupé la prêtrise pour le culte d'Apollon Iètros. Les Spartocides cependant ne limitaient pas leurs activités cultuelles à leur cité de résidence, Panticapée. D’une part, une statue a été consacrée à Kèpoi probablement par une fille du roi Spartokos ${ }^{29}$. D'autre part, Komosarye, épouse du souverain régnant Pairisadès, a fait ériger un groupe statuaire dans le sanctuaire du volcan "Boris et Gleb» ${ }^{30}$. Cela prouve que les membres de la dynastie ont réalisé des donations prestigieuses qui dépassaient le cadre local de la capitale du royaume. Sur la péninsule de Taman, de même, les élites locales étaient actives au sein de leurs cités et à l'échelon régional, comme le prouvent une consécration de Phanagorie faite par Kassalia, fille de Posios, ${ }^{31}$ et une autre consécration de Xenokleidès, un fils du même Posios, à peu près contemporaine, sur le volcan «Boris et Gleb» ${ }^{32}$.

Certains dédicants, par leurs consécrations, entendaient surtout démontrer leur richesse. Ainsi Theopropidès, fils de Mégaclès, qui a consacré la porte d'entrée d'un lieu de culte de Nymphaion, revêtait dans le même temps la charge d'agonothète pour Dionysos ${ }^{33}$. Le même Xenokleidès de Phanagorie ${ }^{34}$ a fait construire un temple à Artémis Agrotera sur le volcan «Boris et Gleb» et une inscription le mentionnant est encore gravée sur le chapiteau d'une colonne in antis de Gorgippia ${ }^{35}$. Dans le royaume du Bosphore, les consécrations d'édifice étaient exceptionnelles et démontrent l'appartenance du dédicant à une élite sociale. Elles attestent également la faveur dont jouissait la culture grecque attique, qui se manifeste aussi dans le rétablissement

29. N. I. Sokol'skij, "Svjatilišče Afrodity v Kepakh», p. 116; N. S. Belova, «Posvjatitel'naja nadpis' iz Kep».

30. Voir infra et n. 39.

3I. CIRB Al'bom 972.

32. CIRB Al'bom 1014.

33. O. J. Sokolova, N. A. Pavličenko, "Novaja posvjatitel'naja nadpis' iz Nimfeja", p. 101 ; O. J. Sokolova, «Lučšie nakhodki iz raskopok Nimfeja za poslednie desjat' let", p. 93; I. Ohlerich, Kultorte und Heiligtümer auf dem Gebiet des Bosporanischen Reiches, p. $120-123$.

34. CIRB Al'bom 1014.

35. Longueur: 19,2 cm, largeur minimale, à gauche: $5 \mathrm{~cm}$, maximale, à droite: $9 \mathrm{~cm}$, hauteur: $4 \mathrm{~cm}$ (A. I. Boltunova, "Novye èpigrafičeskie materialy iz Gorgippii»). 
de la charge d'agonothète, non seulement à Nymphaion ${ }^{36}$, mais aussi à Hermonassa ${ }^{37}$.

Ce qui frappe, c'est que, hormis quelques exceptions datant du $\mathrm{IV}^{\mathrm{e}} \mathrm{s}$. av. J.-C., seules étaient honorées des divinités portant un nom grec. Une seule inscription ${ }^{38}$, retrouvée au pied de la salse (volcan de boue) «Boris et Gleb" sur la côte occidentale du liman Akhtanizovskij sur la péninsule de Taman (fig. 2), concerne deux divinités dont le nom n'est nullement grec, Astara et Sanergès ${ }^{39}$. Et il faut attendre le II ${ }^{\mathrm{e}}$ s. av. J.-C., période où les inscriptions votives conservées sont en net recul, pour retrouver, à Panticapée, deux autres divinités non grecques, Ditagoia ${ }^{40}$ et Angissa ${ }^{41}$. C'est à la même époque qu'Aphrodite est honorée pour la première fois à Panticapée de l'épiclèse Ourania Apatouria, typique du Bosphore. Il semble que ce culte, local à l'origine, se soit alors affranchi

36. Voir n. 37.

37. CIRB Al'bom 1039.

38. Sur les découvertes: K. K. Gerc, Arkheologičeskaja topografija Tamanskogo poluostrova, p. 7 ; N. P. Rozanova, "Posvjatitel'nye nadpisi Fanagorii kak istočnik dlja topografii goroda»; B. von Köhne, "Über die Marmorbasis aus der Zeit des bosporanischen Königs Pairisades I»; N. P. Rozanova, "Posvjatitel'naja nadpis' Sanergu i Astarte»; I. D. Marčenko, "K voprosu o kul'takh Aziatskogo Bospora», p. 102; A. P. Ivanova, Skul'ptura i živopis' Bospora, p. 65 sq. avec illustration; V. F. Gajdukevič, Das Bosporanische Reich, p. 214; I. J. Šaub, Kul'ty i religioznye predstavlenija naselenija Bospora VI-IV vekov do n. è., p. 92 sq. et «Kul't velikoj bogini u mestnogo naselenija Severnogo Pričernomor'ja", p. 208; Y. Ustinova, The supreme gods of the bosporan kingdom, p. 51 sq.; A. A. Zavojkin, "Pamjatnik Satira I na Aziatskom Bospore», p. 54.

39. CIRB Al'bom 1015: consécration de Komosarye, fille de Gorgippos et épouse de Pairisadès, aux dieux tout puissants Sanergès et Astare, Pairisadès étant archonte du Bosphore et de Théodosie et roi des Sindes, de tous les Méotes et des Thatéens. On a retrouvé en même temps que l'inscription deux statues très fragmentées, qui se trouvaient peut-être sur le piédestal. Ces deux sculptures passaient pour disparues. La statue féminine et le piédestal sont conservés au musée de l'Ermitage à SaintPétersbourg: I. Ohlerich, Kultorte und Heiligtümer auf dem Gebiet des Bosporanischen Reiches.

40. Ju. G. Vinogradov, «Votivnaja nadpis’ dočeri carja Skilura iz Pantikapeja i problemy istorii Skifii i Bospora vo II v. do n. è.» avec ill.; F. V. Šelov-Kovedjaev ("Proščanie s Ditagojej») propose une nouvelle lecture de l'inscription, d'où le nom Ditagoia serait absent. Il y voit une consécration à une divinité féminine non précisée, qu'il identifie avec Artémis.

4I. CIRB Al'bom 27. 
Fig. 2 - Salse «Boris et Gleb» sur la presqu'île de Taman. Photographie de Svetlana Kokh.

de son berceau ou bien qu'il ait été établi à son tour dans la capitale du royaume.

Même si, notamment dans les inscriptions votives, on mentionne toujours les nombreux groupes ethniques qui, à partir du IV es. av. J.-C., étaient soumis au roi du Bosphore - et qui étaient manifestement parties intégrantes du royaume -, leurs divinités ne jouent, dans les inscriptions votives des $\mathrm{IV}^{\mathrm{e}}$ et III s. av. J.-C., aucun rôle à Panticapée et seulement un rôle marginal sur la péninsule de Taman - et encore uniquement en dehors des centres importants. Cela soulève des questions sur la coexistence des différents groupes ethniques et la diffusion de leurs traditions religieuses.

Une question intéressante, dans cette perspective, est de savoir qui consacrait les offrandes aux divinités. Sur un total de 67 noms de personne figurant dans les inscriptions votives, qui incluent toutes la mention des dédicants, avec leur patronyme, et les personnes honorées selon les circonstances, 58 ont pu faire l'objet d'une enquête précise. Sur ce nombre, quatre seulement ${ }^{42}$, soit moins de $7 \%$, ont été identifiées 
comme non grecques. Trois de ces quatre noms ${ }^{43}$ renvoient à la famille des Spartocides, que l'on considère originaire non pas de Grèce, mais peut-être de Thrace ${ }^{44}$. Le nom féminin de Komosarya ${ }^{45}$, que l'on trouve mentionné sur une inscription votive de la salse "Boris et Gleb", passe aussi pour thrace ${ }^{46}$.

Une inscription de la première moitié du $\mathrm{IV}^{\mathrm{e}}$ s. av. J.-C., retrouvée dans les environs de la ville de Temrjuk, dans l'est de la péninsule de Taman, nous a transmis l'ethnique Skythès ${ }^{47}$. Il est intéressant que l'on ait employé ici un nom de peuple plutôt qu'un nom propre. Le porteur de cet ethnonyme est désigné par ailleurs comme père d'un certain Démarchos - donc de quelqu'un portant un nom grec. C'est presque le seul cas clairement identifié, dans la tradition épigraphique, d'un nom autre que grec porté par quelqu'un n'appartenant pas à la famille régnante dans la tradition épigraphique. Tous les autres noms mentionnés dans les inscriptions votives sont d'origine grecque - pour autant que l'on puisse en juger.

Voici donc le tableau qui se dégage: dans le royaume du Bosphore, les inscriptions votives sont surtout présentes au IV s. av. J.-C. Auparavant, cet usage ne semble pas avoir revêtu une grande importance et par la suite, au III ${ }^{\mathrm{e}}$ et au $\mathrm{II}^{\mathrm{e}}$ s. av. J.-C., sa popularité est en net recul. À de rares exceptions près, les inscriptions votives honorent uniquement des divinités portant un nom grec et les dédicants portent pour la plupart eux aussi un nom grec. Les quelques exceptions attestées ne proviennent

43. Une fois Leukôn: CIRB Al'bom 25; deux fois Pairisadès: CIRB Al'bom 1015, 1041.

44. Sur la similitude entre les noms des Spartocides et ceux de la dynastie, contemporaine, des Odryses: V. F. Gajdukevič, Das Bosporanische Reich, p. 66-68; Ju. G. Vinogradov, «Die historische Entwicklung der Poleis des nördlichen Schwarzmeergebietes im 5. Jahrhundert v. Chr. ", p. 97 et n. 185. Sur la thèse selon laquelle les Spartocides auraient servi comme mercenaires sous le règne des Archéanactides dans le royaume du Bosphore: M. I. Artamonov, "K voprosu o proiskhoždenii bosporskikh Spartokidov», p. 34; L. Zgusta, "Die verschiedenen Bevölkerungsschichten der griechischen Städte des nördlichen Schwarzmeergebietes", p. 160; voir également Ju. G. Vinogradov, «Die historische Entwicklung der Poleis des nördlichen Schwarzmeergebietes im 5. Jahrhundert v. Chr.», p. 97. Les Spartocides sont tenus aussi parfois pour Grecs, Scythes ou encore Méotes: D. B. Šelov, «Istorija», p. 13.

45. CIRB Al'bom 1015.

46. L. Zgusta, Die Personennamen griechischer Städte der nördlichen Schwarzmeerküste, $\$ 564$.

47. CIRB Al'bom 1111. 
pas des villes les plus importantes du Bosphore ou ne remontent pas audelà du $\mathrm{II}^{\mathrm{e}}$ s. av. J.-C. Ce constat soulève une question: les inscriptions votives étaient-elles un moyen de communication religieuse propre à la communauté culturelle grecque? En d'autres termes, étaient-elles utilisées uniquement par les sujets "grecs" du royaume bosporan, qui se distinguaient ainsi nettement, voire consciemment, des autres groupes ethniques?

\section{Rapport entre la situation économique et les consécrations}

On explique en général l'apparition des inscriptions votives, comme le fait qu'elles aient été d'emblée très bien acceptées, par la prospérité économique du royaume du Bosphore, acquise grâce à sa position de principal fournisseur d'Athènes en céréales. Cette prospérité économique se manifestait dans tous les domaines de la société. De nombreuses transformations indiquent que le niveau de vie de la population augmentait. C'est ainsi qu'ont été construits de nouveaux bâtiments richement décorés à usage d'habitation ou à vocation économique dans les grands établissements et dans les zones rurales. Sur un vaste terrain situé sur les pentes mêmes de l'acropole de Panticapée, par exemple, on a édifié un grand édifice à péristyle très richement décoré, considéré comme le palais des Spartocides ${ }^{48}$. Dans l'arrière-pays du royaume sont apparus au $\mathrm{IV}^{\mathrm{e}}$ s. av. J.-C. de grands complexes économiques dotés de petits lieux de culte pour les habitants ou les visiteurs. L'un de ces complexes très riches du point de vue architectural est General'skoe Zapadnoe, situé sur un éperon rocheux abrupt au-dessus de la mer d'Azov (fig. 3), ainsi nommé d'après la baie voisine ${ }^{49}$. Les petites pièces étaient entourées d'une cour dallée, au centre de laquelle se dressait un autel en pierre. Un site analogue a été mis au jour dans la péninsule de Taman ${ }^{50}$. Mais dans le cas

48. V. P. Tolstikov, G. A. Lomtadze, «K voprosu o vremeni osnovanija basilej Spartokidov na akropole Pantikapeja" et sa bibliographie; V. P. Tolstikov, J. N. Kuz'mina, "Ein neuer Baubefund des 4.-3. Jh. v. Chr. im Zentrum von Pantikapaion".

49. A. A. Maslennikov, Sel'skie svjatilišča evropejskogo Bospora, p. 486-488.

50. Sur la tholos de Taman: N. I. Sokol'skij, Tamanskij Tolos i residencija Khrisaliska; J. N. Kuz’mina, "Organizacija ritualov i žertvoprinošenij v èllinskom svjatilišče», notamment p. 98 sq. 
Fig. 3 - Littoral proche du site archéologique de General'skoe Zapadnoe, sur la mer d'Azov. Photographie de Sergej Anaškevič.

de Taman, on imagine que s'élevait dans la cour un bâtiment rond - une tholos. Chacun des deux sites présente dans l'une de ses petites pièces un espace réservé à des manifestations cultuelles. L'existence d'installations utiles à l'agriculture céréalière - meules et cuves de stockage - a également pu être attestée. Ces domaines servaient probablement d'exploitations agricoles et de résidences de campagne pour leurs propriétaires aisés.

Dans presque tous les sanctuaires du royaume ont alors été entrepris des travaux d'agrandis0sement et de transformation, et sont apparus en outre de nombreux lieux de culte nouveaux, d'une richesse de décoration inhabituelle, tant à l'intérieur qu'en dehors des cités bosporanes. L'exemple le plus célèbre est le bâtiment d'une congrégation religieuse à Nymphaion ${ }^{51}$. Cet édifice intéressant se trouvait un peu à

5I. Sur le mobilier retrouvé et les résultats des fouilles: N. L. Grač, "Raskopki Nimfeja i ego nekropolja» et "K kharakteristike ètničeskogo sostava naselenija Nimfeja v VI-V vv. do n. è.». Dans les rapports intermédiaires sont mentionnés des fûts de colonnes et différents autels: N. L. Grač, «Raskopki Nimfeja» et «Nimfej v konce IV-I vv. do n. è.»; O. J. Sokolova, "Raboty nimfejskoj èkspedicii». Sur les étapes de la construction: O. J. Sokolova, "Investigations of an early hellenistic shrine in 


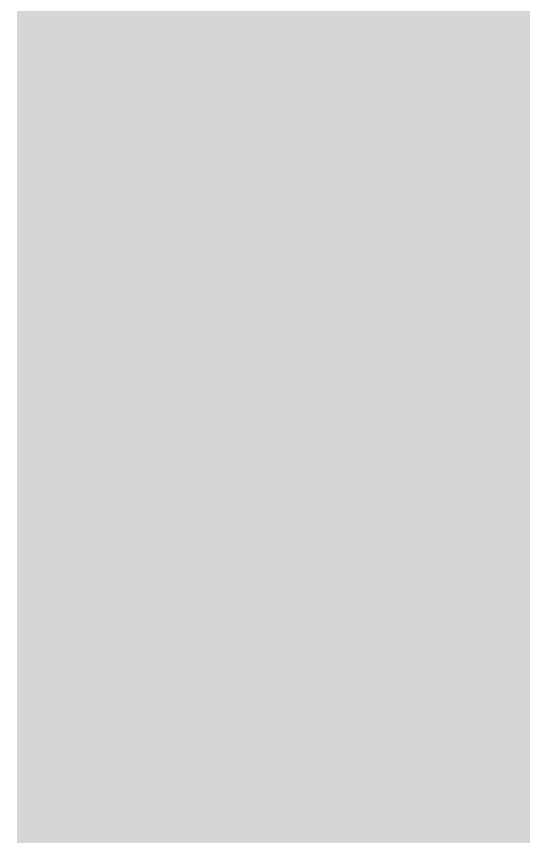

Fig. 4 - Herme d'Hécate aux trois visages, découvert à Panticapée ( $\mathrm{N}^{\circ}$ inv. P.1849-13). (C) Musée d'État de l'Ermitage, Saint-Pétersbourg. Photographie de

Aleksander Lavrentyev.

l'écart de l'ancienne acropole, à flanc de coteau, et s'étageait sur trois terrasses. Il comprenait plusieurs petites pièces reliées par des marches d'escalier et une cour dallée. Des autels de différentes formes se dressaient sur plusieurs niveaux. Un autel de base carrée avec une cupule est encore visible aujourd'hui in situ dans la cour. Le site est surtout célèbre pour ses inscriptions et représentations de bateau gravées ou sculptées dans les stucs des murs ${ }^{52}$.

Le nouveau complexe cultuel de Nymphaion, avec son portique et la consécration de l'Agonothète, qui remonte lui aussi au $I^{\mathrm{e}}$ s. av. J.-C., était directement relié au bâtiment des graffitis par un escalier ${ }^{53}$. Cet ensemble, qui n'est pas encore intégralement publié, comprenait peut-être un théâtre et un lieu de culte pour Dionysos, avec de la place pour les concours musicaux en l'honneur du dieu. À Panticapée, dans une grotte naturelle située à proximité immédiate de la porte ouest de l'enceinte de la ville, est apparu au cours du IV e s. av. J.-C. un lieu de culte pour Hécate ${ }^{54}$. Les parois rocheuses de la grotte

Nymphaeum» et "Nymphaeum», p. 772 sq.; A. Wąsowicz, «Nymphaion», p. 162 sq. En outre: L. P. Gagen, L. S. Gavrilenko, "The study and restoration of antique fresco from Nymphaeum »; E. A. Molev, Bospor v period ellinizma, p. 7; J. S. Morrison, Greek and Roman oared worships, p. 207-214; N. A. Frolova, Die frühe Münzprägung vom Kimmerischen Bosporos, p. 56.

52. O. Höckmann, «Naval and other graffiti from Nymphaion» et sa bibliographie.

53. O. J. Sokolova, «Nymphaeum» et sa bibliographie. Pour le bateau «Isis», voir P. Burgunder, C. Semenzato, «Retour à Alexandrie».

54. V. D. Blavatskij, "Materialy po istorii Pantikapeja», p. 22 et plan ill. 6 p. 14 $\mathrm{n}^{\mathrm{o}} 18$. 
Fig. 5 - La montagne de Mai («Majskaja gora»). Photographie de Sergej Bezuglov.

étaient recouvertes de plaques de marbre. Il s'y dressait probablement une borne triangulaire, un Hékateion, identifié il y a quelques années dans les collections de l'Ermitage à Saint-Pétersbourg ${ }^{55}$ (fig. 4). Dans la sous-colonie de Myrmékion, au nord de Panticapée, toujours durant le $\mathrm{IV}^{\mathrm{e}}$ s. av. J.-C., fut érigé - là aussi un peu à l'écart du centre du bourg un bâtiment de petites dimensions avec plusieurs autels, interprété comme un sanctuaire de Déméter.

D'autres sanctuaires, liés à des particularités géographiques et qui se distinguaient par une architecture à vrai dire fort modeste, ont subi au $\mathrm{IV}^{\mathrm{e}}$ s. av. J.-C. des modifications visant à enrichir leur décoration. C'est ainsi qu'un sanctuaire sur la montagne de Mai ("Majskaja gora») - une salse aux portes de la colonie de Phanagorie sur la péninsule de Taman ${ }^{56}$ - a été pourvu d'un bâtiment en pierre (fig. 5). Ce qui est remarquable dans ce sanctuaire, c'est que pour le reste il a conservé,

55. L. I. Akimova, «Novyj pamjatnik skul'ptury iz Pantikapeja», p. 84 n. 139. Dans le catalogue des sculptures de l'Ermitage dressé par $\mathrm{O}$. Waldhauer, cette pièce est répertoriée avec la mention "origine inconnue»: O. Waldhauer, Die Antiken Skulpturen der Ermitage, p. $24 \mathrm{n}^{\circ} 259$ et table XVII.

56. Sur les découvertes du sanctuaire de Phanagorie en général: J. N. Kuz’mina, «Sakral'nye kompleksy Fanagorii VI-I vv. do n. è.». 
à peu de choses près, son état architectural d'origine. Lors de fouilles menées dans les années 1970, on a retrouvé dans une coulée de boue des centaines de statuettes en terre cuite, probablement jetées dans la cheminée du volcan par les participants au culte et qu'une éruption avait restituées. Les statuettes représentaient toutes des sujets féminins. Il s'agit pour la plupart de protomés.

Il existait sur les flancs de l'ancienne acropole de Nymphaion un sanctuaire naturel alimenté en eau douce par une source et qui ne présentait jusqu'au IV ${ }^{\mathrm{e}}$ s. av. J.-C. qu'une architecture extrêmement modeste. Plusieurs murs servaient à stabiliser le terrain en pente et à délimiter le sanctuaire. Et c'est seulement lorsque le royaume a connu la prospérité économique qu'un petit édifice a été rajouté ${ }^{57}$.

Cette période de prospérité est marquée en outre par l'édification de kourganes au mobilier funéraire très riche, dont celui de Koul-Oba près de Kertch ${ }^{58}$. Ces grands ensembles funéraires étaient placés bien en vue le long des voies de communication qui desservaient les cités du Bosphore. Certains d'entre eux étaient dotés d'un précieux mobilier qui témoigne de la richesse des défunts qui y sont inhumés.

Incontestablement, l'apparition et le succès des inscriptions votives au $\mathrm{IV}^{\mathrm{e}}$ s. av. J.-C. résultent aussi de la puissance économique accrue des dédicants. Un coup d'œil sur les découvertes datant d'avant ou d'après l'essor de la région montre toutefois que la situation économique n'explique pas tout. Avant même l'établissement de relations commerciales avec Athènes, en effet, il existait sans nul doute dans le royaume du Bosphore des élites qui pouvaient se permettre d'offrir des inscriptions votives.

57. I. Ohlerich, Kultorte und Heiligtümer auf dem Gebiet des Bosporanischen Reiches, p. 110-121, et sa bibliographie. Les fouilles ont été conduites de 1939 jusque dans les années 1940 par la mission archéologique de Nymphaion détachée par l'Ermitage de Saint-Pétersbourg, sous la direction de M. M. Khudjak. Dans ses publications, Khudjak rapporte l'existence, à proximité du lieu de culte, de deux grottes ou cavernes dans la roche, où, selon lui, devait se trouver une source, utilisée ensuite pour les besoins $\mathrm{du}$ culte: entre autres, M. M. Khudjak, "Predvaritel'nye itogi raskopok poslednikh let v Nimfee", p. 84; et aussi A. X. Kocybala, Greek Colonization on the North Shore of the Black Sea in the Archaic Period, p. 298; I. J. Šaub, Kul'ty i religioznye predstavlenija naselenija Bospora VI-IV vekov do n. è., p. 106. Ces grottes aujourd'hui ne sont plus reconnaissables. En outre, apparemment, on n'y a trouvé aucun objet remontant à l'Antiquité et susceptible d'appuyer cette hypothèse.

58. J. M. Paromov, «Kurgannyj nekropol’ Germonassy», p. 206, ill. 1. 


\section{Avant la phase de prospérité}

Les cités du royaume ont été d'abord fondées comme des poleis autonomes et chacune possédait en tant que telle des lieux de culte propres. De tels lieux de culte, propres à la polis, sont attestés d'une part à Panticapée - la future capitale du royaume -, d'autre part à Nymphaion, quelques kilomètres plus au sud. Les deux cités se sont constituées à la fin du $\mathrm{VI}^{\mathrm{e}}$ s. av. J.-C. selon un plan semblable: les habitations sont groupées tout autour d'un centre religieux situé sur une éminence qui occupe une position centrale.

À Panticapée, dès la fin du VI ${ }^{\mathrm{e}}$ s. av. J.-C., on a construit un monumental temple d'ordre ionique dont il reste quelques tambours et bases de colonnes, et des fragments d'entablement. On suppose qu'il se dressait sur le premier replat du mont Mithridate qui s'élève au centreville ${ }^{59}$. Sur cette même hauteur, se trouvait à la fin du VIe s. av. J.-C. un complexe constitué d'une tholos et d'un bâtiment de plusieurs pièces, qui tranchait nettement, par sa situation et son équipement comme par sa taille et son style de construction, avec les bâtiments d'habitation courants alors à Panticapée ${ }^{60}$.

On peut tirer des conclusions semblables pour Nymphaion à la même époque. Sur l'éminence qui s'élève au centre de l'établissement, on a construit à la fin du VI ${ }^{\mathrm{e}}$ s. av. J.-C. un grand bâtiment avec une abside intérieure et une rangée de colonnes centrale ${ }^{61}$, dont la fonction n'est pas claire. Il fait penser aux premiers édifices qui servaient aux assemblées ou au culte des congrégations religieuses. À proximité immédiate - comme à Panticapée -, se trouvait un bâtiment de plusieurs pièces.

Quelle était la fonction de ces constructions? Un coup d'œil à leur histoire montre bien qu'ils ont été tous deux totalement détruits après une période d'utilisation relativement courte. Le lieu de culte de Nymphaion a été recouvert par la nouvelle enceinte de la ville dans la première moitié du $\mathrm{IV}^{\mathrm{e}} \mathrm{s}$. av. J.-C. et, partant, clairement et entièrement sécularisé pour tous ceux qui le fréquentaient. À Panticapée, on a créé

59. Pour un exposé détaillé: V. P. Tolstikov, «Khram Apollona na akropole Pantikapeja".

6o. V. P. Tolstikov, «Pantikapaeum», p. 717-719.

6I. Sur ce sujet, y compris les constructions antérieures: M. M. Khudjak, Iz istorii Nimfeja VI-III vekov do n. è., p. 12-24; O. J. Sokolova, "Nymphaeum», p. 765 sq. 
au bout de quelques années un nouveau complexe comprenant un bâtiment de plusieurs pièces, considéré comme le palais des Spartocides, ainsi qu'un temple in antis situé dans une cour attenante ceinte d'un mur ${ }^{62}$. Ce nouvel ensemble a vraisemblablement été érigé pour prolonger l'utilisation précédente du site. Peut-être des élites locales, détentrices de charges religieuses, ont-elles résidé là avant la destruction et réuni une communauté cultuelle - de colons - dans le bâtiment de culte tout proche. La destruction et la transformation coïncident avec la prise de pouvoir des Spartocides et la création d'un vaste État. Ce moment historique a probablement été, sinon provoqué, du moins accompagné de luttes entre les protagonistes, avec pour conséquence la destruction des sanctuaires et des résidences liés aux groupes au pouvoir.

Par leur riche décoration et leur plan généreux, ces sites tranchent nettement avec les habitations de l'époque. Mais apparemment, dans cette première phase de la colonisation, on ne voyait pas d'intérêt à se présenter comme individu en fournissant des informations personnelles sur soi-même et sur sa religion. Probablement les groupes religieux étaient-ils encore peu fournis; leurs membres se connaissaient et n'avaient donc pas besoin de proclamer leur piété par écrit, hors de leur cercle.

\section{Après la phase de prospérité}

On peut faire un constat semblable pour la période qui suit la crise du $\mathrm{II}^{\mathrm{e}}$ s. av. J.-C. S’affirme alors une élite économique, qui se manifeste avant tout par la construction d'imposantes résidences somptueusement décorées sur le flanc nord du mont Mithridate à Kertch ${ }^{63}$. Ces résidences, pour autant qu'on puisse en juger, étaient conçues comme des maisons à péristyle avec une cour dallée. À proximité immédiate de ce qui est sans doute la plus magnifique d'entre elles - dite "palais des Spartocides", déjà mentionnée - se trouvait un temenos avec un temple in antis. Cet ensemble était situé à l'intérieur du deuxième mur d'enceinte de l'acropole, ce qui le rendait sûrement moins accessible. Un peu plus au nord se trouvait un autre édifice à péristyle, dont la cour dallée indique

62. V. P. Tolstikov, «Pantikapaeum», p. 730-734 et sa bibliographie.

63. I. D. Marčenko, «Raskopki zdanija II v. do. n. è. v Pantikapee 1963-1965». 
la présence d'un autel aménagé dans le dallage ${ }^{64}$. Ces deux lieux de culte présentent deux modalités d'exclusivité religieuse, caractérisée par l'accessibilité et la délimitation. Dans les deux cas, l'usage de ces lieux de culte était réservé à un cercle restreint de personnes - les habitants de la maison ou les résidents du flanc nord de l'acropole? Une telle exclusivité rend superfétatoires les formes de piété fixées dans des inscriptions, car les participants au culte se connaissaient et les personnes étrangères n'avaient qu'un accès limité au site. C'est pourquoi dans le complexe cultuel de General'skoe Vostočnoe ${ }^{65}$, édifié au II ${ }^{\mathrm{e}}$ s. av. J.-C. sur le littoral azovien de la péninsule de Kertch, on n'a pas exposé non plus d'inscriptions votives. Constitué d'une rangée de plusieurs petits édifices cultuels, ce site, rien que par son emplacement et son équipement, était prévu uniquement pour de petits groupes religieux.

La consécration à Ditagoia ${ }^{66}$, provenant de Panticapée et datant du $\mathrm{II}^{\mathrm{e}}$ s. av. J.-C., représente un exemple opposé. Cette inscription était portée dans la montée nord de l'acropole de Panticapée sur une table sacrificielle dressée dans une niche ouverte. Par sa position et sa forme architecturale, elle était accessible à un public plus large. En outre, la dédicante était la fille du roi des Scythes, Skilouros, originaire de Neapolis Scythica, au centre de la Crimée. Elle n'appartenait probablement à aucune des communautés religieuses existantes, du moins au début. En exprimant publiquement sa piété, d'une part, elle se présente aux autres participants du culte, d'autre part elle déclare son appartenance à un groupe religieux et social. Dans cette perspective, la consécration d'une table, pour anachronique qu'elle puisse apparaître à première vue dans une période marquée par un faible intérêt pour les marques de piété publiques, est tout à fait sensée.

\footnotetext{
64. Sur ledit Prytanée: I. Ohlerich, Kultorte und Heiligtümer auf dem Gebiet des Bosporanischen Reiches, p. 84-86.

65. A. A. Maslennikov, Sel'skie svjatilišča evropejskogo Bospora, p. 89 sq.

66. Voir la nouvelle proposition de lecture de l'inscription établie par F. V. ŠelovKovedjaev ("Proščanie s Ditagojej»).
} 
Reconnaissance suprarégionale des élites

La fusion des différentes colonies pour constituer un vaste État au tournant des $\mathrm{V}^{\mathrm{e}}$ et $\mathrm{IV}^{\mathrm{e}}$ s. av. J.-C. a engendré une situation sociotopographique totalement nouvelle. Désormais les colonies n'étaient plus seulement connectées les unes aux autres, mais se présentaient comme un royaume soudé et un partenaire commercial dans tout l'espace méditerranéen. Les élites locales, dont le rayon d'action se limitait précédemment à leur environnement social immédiat, faisaient face à un nouveau défi: se présenter avec leur statut au-delà de leurs anciennes frontières. Pour ce faire, les inscriptions votives étaient un moyen éprouvé: elles étaient financièrement abordables, mobiles et communément compréhensibles grâce à leur formule simple. Les consécrations prestigieuses et coûteuses comme celles des Agonothètes ou les fondations de monuments étaient une exception. Il ne s'agissait donc pas tellement pour les donateurs de montrer leur richesse, mais bien plutôt de se rattacher publiquement à un groupe social ou religieux actif au-delà des environs. Ces consécrations ont aussi modifié la communication religieuse: elles ont en partie remplacé le contact direct avec les coreligionnaires, permis de dépasser les frontières géographiques et architecturales et de constituer de nouvelles communautés cultuelles. L'exclusivité n'est plus limitée au lieu d'exposition; les inscriptions excluent plutôt ceux qui ne comprennent pas cette forme de communication - par exemple, ceux qui ne maîtrisent pas le grec ou ne savent pas le lire.

C'est peut-être aussi ce qui explique la distribution inégale des inscriptions votives entre les centres du royaume. Elles n'étaient pas exposées là où vivaient les dédicants, mais à une échelle suprarégionale. C'est ainsi que Komosarya, fille de Gorgippos et femme du souverain régnant Pairisadès, a consacré deux statues sur la salse "Boris et Gleb ». Xenokleidès, qui y a fondé là un temple, était le fils de Posios de Phanagorie. Par ce biais, ils se faisaient connaître à un large public bien au-delà de leur cité.

Les habitants du Bosphore voyaient donc surtout dans ces consécrations, semble-t-il, un moyen de se représenter dans un cadre géographique et socioreligieux plus vaste, là où la communauté cultuelle ne se limitait pas à quelques participants, là où l'on entendait atteindre un public plus large et toucher ou établir une communauté par-delà la topographie. C'était aussi le but poursuivi par les souverains du Bosphore lorsqu'ils 
ont fait exposer des consécrations et des hommages dans la cité sinde de Semibratnee ${ }^{67}$, à Delphes et à Délos ${ }^{68}$. Par cette marque de piété individuelle, ils se joignaient à une communauté cultuelle plus grande.

\section{Culture-groupe-piété: à propos de la question de l'acculturation}

Les inscriptions votives ne sont qu'une partie de la vie religieuse et cultuelle des habitants du royaume du Bosphore. Eu égard à la brièveté de leur floraison et à leur nombre relativement restreint d'inscriptions conservées, il est difficile de tirer de leur analyse des conclusions valables pour l'ensemble de la région. Elles représentent néanmoins les expressions religieuses individuelles, conservent le nom des divinités honorées et des participants à leur culte et sont aussi, justement pour cette raison, un témoignage important sur la manière dont fonctionnait la coexistence interculturelle entre les habitants du Bosphore. L'absence quasi totale d'éléments non grecs n'en est que plus étonnante à première vue. Surtout dans la première phase des consécrations, les inscriptions sont presque exclusivement adressées à des divinités grecques par des dédicants portants des noms grecs. C'est particulièrement vrai dans la capitale, Panticapée. Sur la péninsule de Taman, notamment dans les sanctuaires extra-urbains ou dans les grands sanctuaires situés en dehors des colonies, par exemple celui sur la salse «Boris et Gleb", des divinités non grecques étaient honorées dès le $\mathrm{IV}^{\mathrm{e}} \mathrm{s}$. av. J.-C.

Mais peut-on conclure de là que les colonies grecques n'étaient habitées que par des Grecs, ou que les non-Grecs ne participaient pas aux cérémonies cultuelles typiquement grecques, ni ne manifestaient leurs conceptions religieuses? Ou bien qu'ils étaient entièrement assimilés à la culture grecque? Ces questions sont particulièrement intéressantes dans la perspective de la coexistence des différentes communautés culturelles au sein du royaume du Bosphore.

On a proposé plusieurs explications. Certains chercheurs sont d'avis que, derrière les noms grecs figurant dans les inscriptions, se cachent des divinités non grecques et que l'on identifiait les divinités non grecques

67. V. P. Jajlenko, "Votiv Levkona I iz Labrisa».

68. B. Hintzen-Bohlen, Herrscherrepräsentation im Hellenismus, p. 192, 179, 216 sq., n 5 f2; B. Schmidt-Dounas, Geschenke erhalten die Freundschaft, p. 146 et 157. 
et leurs fonctions à celles des divinités grecques ${ }^{69}$, comme on a pu le démontrer pour Apollon Iètros et Aphrodite Ourania. D'autres imaginent plutôt que les communautés culturelles vivaient les unes à côté des autres et que les Grecs honoraient exclusivement les divinités qu'ils avaient importées alors que les autres habitants (non grecs) n'ont que très légèrement adapté leur culte à l'aune du modèle grec ${ }^{70}$.

Un coup d'œil au mobilier retrouvé sur les lieux de culte ainsi qu'aux autres vestiges matériels du royaume du Bosphore permet de mieux cerner le phénomène. Une chose frappe quand on fait la comparaison avec les sanctuaires grecs ou avec les rares trouvailles d'origine scythe: l'influence des conceptions religieuses grecques est pratiquement la seule que l'on constate, non seulement dans les inscriptions votives, mais aussi dans les sites cultuels du Bosphore. Les premières acropoles décrites plus haut et les domaines agricoles de l'arrière-pays des deux péninsules n'ont rien d'inhabituel pour des sites grecs. Les sanctuaires naturels comme la montagne de Mai près de Phanagorie et le sanctuaire de Déméter à Nymphaion présentent des éléments architecturaux adaptés au site et à leur fonction et qui ne sont pas caractéristiques d'une ethnie, tandis que le petit mobilier est de style grec. Les sites cultuels tardifs de la péninsule de Kertch sont certes insolites, mais ne peuvent être classés ni comme grecs, ni comme scythes, ni être rattachés à une autre culture du PontEuxin septentrional.

Les amoncellements de cendre (eschara)

Seuls les nombreux monticules de cendre (désignés en grec sous le terme de escharalė $\sigma \chi \alpha ́ \rho \alpha)$, attestés sur la péninsule de Kertch depuis le $\mathrm{VI}^{\mathrm{e}} \mathrm{s}$.

69. M. Rostovtzeff, "Le culte de la Grande Déesse dans la Russie Méridionale» et Iranians and Greeks in South Russia; M. I. Rostovcev, "Iranskij konnyj bog i Jug Rossii"; I. J. Šaub, Kul'ty i religioznye predstavlenija naselenija Bospora VI-IV vekov do n. è., en part. p. 53-56 et 117-121 ; L. P. Kharko, "Kul't Afrodity na Bospore Kimmerijskom»; D. B. Šelov, "K voprosu o vzaimodejstvii grečeskikh i mestnykh kul'tov v Severnom Pričernomor'e»; N. I. Sokol'skij, «Svjatilišče Afrodity v Kepakh», p. 116; A. P. Iwanowa, "Gestalten der örtlichen Mythologie in der Kunst des Bosporos"; Y. Ustinova, "Aphrodite Ourania of the Bosporus" et The supreme gods of the bosporan kingdom.

70. I. A. Emec, Greko-Varvarskie religioznye vzaimovlijanija na Bospore Kimmerijskom. 
av. J.-C., peuvent être clairement rattachés aux populations indigènes du nord de la mer Noire. De typologie variée et présents dans différents contextes urbains, ces monticules de cendre ${ }^{71}$ sont fréquents dans le royaume du Bosphore. Ils sont présents dans les colonies de Panticapée ${ }^{72}$ et de Nymphaion ${ }^{73}$, dans les sous-colonies de Kytaia ${ }^{74}$ au sud de la péninsule de Kertch et de Myrmèkion ${ }^{75}$, comme dans de nombreuses implantations de l'arrière-pays. Il s'agit de couches de cendre stratifiées plus ou moins importantes mêlées à de la terre et de la glaise, qui peuvent atteindre plusieurs mètres de hauteur sur une surface de $1000 \mathrm{~m}^{276}$. La taille énorme de ces amoncellements et le mobilier que l'on y a retrouvé témoignent qu'ils ont été utilisés, ou qu'ils se sont formés, pendant des décennies, si ce n'est des siècles. Certains de ces monticules de cendre se trouvent dans des centres urbains ${ }^{77}$, d'autres plutôt à leur périphérie ou bien en-dehors ${ }^{78}$. Certains sont situés sur le sol d'origine, d'autres à l'emplacement d'anciennes structures architecturales détruites ${ }^{79}$.

On trouve des monticules de cendre analogues à ceux du Bosphore dans tout l'espace s'étendant au nord du Pont-Euxin. Ils sont partie intégrante de la culture des diverses populations de cette région et les chercheurs ont même baptisé l'une d'entre elles d'après ce phénomène ${ }^{80}$. Ces monticules sont composés en général d'un conglomérat de cendre, d'os d'animaux et de petits fragments de poterie - provenant entre autres de statuettes en terre cuite. Les spéculations sur la signification et la fonction de ces lieux de culte sont allées bon train. À la différence des autels

7I. Pour une présentation détaillée des monticules de cendre du Bosphore: I. Ohlerich, Kultorte und Heiligtümer auf dem Gebiet des Bosporanischen Reiches, p. 222270.

72. V. N. Zin'ko, «Bosporskij gorod Nimfej i varvary».

73. I. Ohlerich, Kultorte und Heiligtümer auf dem Gebiet des Bosporanischen Reiches, p. 128 sq.

74. N. V. Moleva, Očerki sakral'noj žizni Bospora et «Kitejskoe svjatilišče».

75. V. F. Gajdukevič, «Raboty na Bospore», p. 101 ; A. M. Butjagin, «Novye issledovanija rannego mirmekijskogo zol'nika».

76. Comme p. ex. l'eschara de Kytaia: N. V. Moleva, Očerki sakral'noj žizni Bospora.

77. Tel celui de Nymphaion: voir n. 73.

78. Ainsi les nombreux monticules de cendre des implantations rurales dans l'arrièrepays de la péninsule de Kertch, p. ex. les quatre qui entourent le site d'Artezian: A. A. Maslennikov, Èllinskaja khora na kraju Ojkumeny, p. 120 sq. avec ill. 77.

79. Nymphaion et Kytaia.

80. Dans la culture de la steppe arborée, que l'on identifie avec celle des premiers Scythes: I. I. Ljapuškin, «Dneprovskoe lesostepnoe levobereže v èpokhu železa». 
sacrificiels de Grèce, le petit mobilier et les os provenant des monticules de cendre ne présentent aucune trace de calcination ${ }^{81}-$ il ne s'agit donc pas d'autels sacrificiels où l'on aurait accompli des actes cultuels et abandonné les restes du sacrifice. En outre, ils sont placés librement dans les cités, sur des terrains non délimités, et ne semblent accompagnés d'aucun bâtiment. Ce ne sont donc pas non plus des restes de sacrifices au sens grec. On y collectait plutôt des déchets issus de différentes activités. Dans certains de ces monticules de cendre étaient aménagées des fosses contenant des os d'animaux, qui font penser, par leur composition, à des restes de sacrifices - souvent un mélange de crânes de chevaux ou de chiens et d'autres os d'animaux. De par sa nature, le mobilier retrouvé dans les couches superficielles environnantes correspond à celui retrouvé dans les zones d'habitation contemporaines. Cela laisse supposer que ce matériel était éliminé des habitations avec la cendre du foyer pour être déposé sur les monticules, et qu'il résulte d'un culte lié à la sphère domestique. Dans sa description du panthéon scythe, Hérodote mentionne Tabiti comme divinité principale ${ }^{82}$. Il en fait l'équivalent de Hestia, la protectrice grecque du foyer domestique. Les immenses monticules de cendre pourraient lui avoir été dédiés; par leur taille et leur étendue, ils symbolisaient à la fois la cohésion et le nombre des participants au culte, et la longévité de leur existence et de leur utilisation.

Ces lieux de culte caractérisent de nombreux établissements du Bosphore dès le VI $\mathrm{e}^{\mathrm{e}}$ s. av. J.-C. À partir du IVe s. av. J.-C. au plus tard, on en trouve aussi dans les grandes colonies comme Panticapée et Nymphaion, où un immense monticule de cendre recouvrait le complexe avec la salle des graffitis et le portique de Dionysos. Du point de vue religieux et architectural, ils constituaient un contrepoint aux lieux de culte grecs, où l'on exposait des inscriptions votives et où les participants se représentaient eux-mêmes comme individus avec leur piété et leur statut. Soutenus par une communauté entière, ils sont, comme les kourganes, le résultat et le témoignage d'années d'activités cultuelles - l'immense marque de piété de toute une communauté.

8I. Pour une présentation détaillée de la nature du mobilier retrouvé et de la stratification: I. Ohlerich, Kultorte und Heiligtümer auf dem Gebiet des Bosporanischen Reiches, p. 243-249.

82. Hérod. IV.59 et IV.68. 


\section{Les interactions ethniques au miroir des nécropoles}

Les contacts intensifs ${ }^{83}$ entre les cultures grecques et celles du PontEuxin septentrional se sont manifestés tout particulièrement dans les rituels funéraires observés dans les cités les plus importantes ${ }^{84}$. Le long des axes desservant les villes s'étendaient des nécropoles à sépultures en pleine terre, ainsi que des nécropoles tumulaires, constituées parfois de centaines de kourganes ${ }^{85}$, qui marquaient fortement, et continuent de marquer de nos jours, la topographie religieuse de la région. Rien qu'à leur emplacement, bien "grec», on voit ce mélange d'éléments divers. La disposition des kourganes, la manière d'inhumer et le mobilier funéraire comptent parmi les spécificités de la culture du Bosphore. Plusieurs travaux ont essayé de classer les tombes en fonction des groupes sociaux et ethniques, afin de pouvoir caractériser plus précisément la coexistence des différentes cultures ${ }^{86}$. Ce n'est pas le lieu ici de développer chaque argument. Ce qui est le plus important, c'est de constater que l'existence de différents groupes ethniques sur la péninsule de Kertch et de Taman se retrouve dans la diversité des rituels funéraires des nécropoles des grandes villes ${ }^{87}$. Dans la nécropole tumulaire de Panticapée, on trouve notamment beaucoup d'éléments qui renvoient aux traditions scythes. Le kourgane de Koul'-Oba, en général interprété comme tombeau d'un aristocrate scythe, présente un nombre considérable de traditions funéraires scythes. Ainsi le défunt est-il inhumé avec sa femme, il y a des

83. Par exemple, les interventions des Scythes dans les querelles de succession dynastique dans le Bosphore (310-309 av. J.-C.) : A. N. Ščeglov, «O greko-varvarskikh vzaimodejstvijakh na periferii èllinističeskogo mira».

84. E. G. Kastanajan, «Gruntovye nekropoli bosporskikh gorodov i mestnye ikh osobennosti»; V. F. Gajdukevič, «Nekropoli nekotorykh bosporskikh gorodov».

85. Récemment, Ja. M. Paromov s'est efforcé de reconstituer la topographie des nécropoles à kourganes sur la péninsule de Taman. Dans ses articles sur les nécropoles de Hermonassa et Kèpoi, parus en 2002 et 2003 dans les Drevnosti Bospora, il a publié des plans qui mettent en évidence la position privilégiée des kourganes le long des axes de communication: J. M. Paromov, «Kurgannyj nekropol' Germonassy», p. 206, ill. 1.

86. A. A. Maslennikov, Naselenie Bosporskogo Gosudarstva v VI-II vv. do. n. è., p. 193 sq., avec la bibliographie sur le sujet.

87. Les considérations qui suivent sont fondées sur l'ouvrage de A. A. Maslennikov (Naselenie Bosporskogo Gosudarstva v VI-II vv. do. n. è., p. 54-63), qui traite et interprète les exemples les plus importants. 
indices de sacrifices d'animaux et un grand nombre de pièces d'armes ${ }^{88}$. En tout, selon le calcul de G. A. Cvetaeva, les éléments scythes constituent un quart des rituels funéraires dans la nécropole tumulaire de Panticapée. Sont en outre attestés des éléments sindes et sauromatosarmates ${ }^{89}$. Dans la nécropole à sépultures en pleine terre de Panticapée, en revanche, on ne trouve que peu de tombes présentant des éléments non grecs - principalement sindes et méotes ${ }^{90}$. Les traditions funéraires scythes marquent également de leur sceau les kourganes du groupe des Trois frères dans la nécropole tumulaire de la ville de Nymphaion, marquée dès le $\mathrm{V}^{\mathrm{e}}$ s. av. J.-C. par la culture scythe. Pour cette époque, on y décèle aussi des éléments sindes ${ }^{91}$. La nécropole terrestre de Nymphaion, en revanche, n'a encore guère fait l'objet de recherches sur ce point et n'a été publiée que dans des rapports provisoires ${ }^{92}$.

\section{Onomastique et composition ethnique}

Hormis les inscriptions votives, le reste de la tradition épigraphique apporte aussi des indications sur les mécanismes du mélange des populations. Pour la recherche qui suit, nous avons procédé à une étude statistique des inscriptions funéraires et des listes de noms de la région du royaume du Bosphore ${ }^{93}$. Sur les 818 noms comptés, 723 étaient lisibles et identifiables. Sur ces 723 noms, 675 ont pu être identifiés comme

88. Ibid., p. 54.

89. Une tombe à kourgane du IV e s. av. J.-C. sur la montagne Temir («TemirGora»), près de Panticapée, présente des éléments qu'E. V. Jakovenko considère comme sauromato-sarmates: E. V. Jakovenko, «Kurgan Temir-Gora»; V. D. Blavatskij, «Ob ètničeskom sostave Pantikapeja v IV-III vv. do n. è.»; A. A. Maslennikov, Naselenie Bosporskogo Gosudarstva v VI-II vv. do. n. è., p. 55; voir aussi V. D. Blavatskij, "O proiskhoždenii bosporskikh sklepov s ustupčatymi perekrytijami».

90. Sur les 251 tombes en pleine terre analysées, seules 27 présentent des éléments non grecs, que l'on attribue à des communautés sindes ou méotes: A. A. Maslennikov, Naselenie Bosporskogo Gosudarstva v VI-II vv. do. n. è., p. 51.

9I. L. F. Silant'eva, "Nekropol' Nimfeja»; A. A. Maslennikov, Naselenie Bosporskogo Gosudarstva v VI-II vv. do. n. è., p. 56; V. N. Zin’ko, "Bosporkij gorod Nimfej i varvary", p. 210.

92. A. A. Maslennikov, Naselenie Bosporskogo Gosudarstva v VI-II vv. do. n. è., p. 50. Pour l'attribution du kourgane de Temir-Gora plutôt aux Scythes: E. V. Jakovenko, «Kurgan Temir-Gora».

93. I. Ohlerich, Kultorte und Heiligtümer auf dem Gebiet des Bosporanischen Reiches, Annexe 4. 
grecs, 11 comme thraces, 18 comme iraniens et deux comme perses. En outre, les ethnonymes «sindes» et «scythes» sont employés 17 fois. Avec $93,75 \%$ du total, les noms grecs se taillent donc dans ces sources la part du lion. Les noms autres que grecs ne représentent que 6,25\%. La proportion relative des noms grecs et autres n'y diffère donc pas sensiblement de celle des inscriptions votives - où la part des premiers est à peine plus petite $(93,1 \%)$. La différence est plus marquée pour ce qui est de l'origine des noms autres que grecs. Alors que dans les inscriptions votives ces noms propres sont tous d'origine thrace (à l'exception d'un seul ethnonyme) et peuvent être tous sans exception rattachés à la famille régnante, en revanche, dans les inscriptions funéraires et les listes de noms, nous avons identifié, outre des noms thraces, d'autres noms iraniens et perses. L'éventail est donc nettement plus large et atteste l'existence de tous ces groupes culturels dans la région du royaume du Bosphore.

\section{Conclusion}

Notre recherche a montré que l'influence des idées et des modes de vie non grecs était variable selon les domaines. Si on reprend les sources présentées plus haut pour établir les structures ethniques dans le royaume du Bosphore, alors il semble que les cultures aient vécu les unes à côté des autres et que seuls les non-Grecs aient montré une volonté d'échange. Éléments en soi non grecs, les monticules de cendre et les kourganes présentent pourtant des caractères grecs, alors que, pour le reste, les sanctuaires, les consécrations et les nécropoles à sépultures en pleine terre n'ont guère subi de modifications.

Toutefois, il y a des indices que les rapports entre les différentes populations étaient plus nuancés que l'image fournie à première vue par les sources. Ainsi les noms figurant dans les inscriptions ne permettent-ils sans doute pas de conclure unilatéralement à l'appartenance ethnique des individus qui les portent. Les membres de la dynastie des Spartocides portaient tant des noms grecs que thraces. Au IV s. av. J.-C. nous est transmis le nom d'un roi sinde, Hécatée, dont le père portait le nom barbare d'Octamasadès ${ }^{94}$. Une même liberté dans le choix des

94. Dans une inscription de la cité de Semibratnee, l'antique Labrys: voir T. V. Blavatskaja, "Posvjaščenie Levkona I»; V. P. Jajlenko, "Votiv Levkona I iz 
noms ressort également des inscriptions conservées. Dans une liste de Gorgippia ${ }^{55}$ (qui mentionne le nom et le patronyme de citoyens de la ville) figurent par exemple un certain Sindos, fils de Démétrios ${ }^{96}$ ou bien un Aristoclès, fils d'un Skythès ${ }^{97}$ ou encore un Maisès, nom iranien, fils d'un Calligénès, nom grec ${ }^{98}$. Apparemment, le nom était donc moins tributaire de l'origine génétique que du choix de son détenteur (ou de ses parents) et ne reflète donc pas du tout la réelle origine ethnique de celui-ci. Cela dit, il convient de souligner que les noms grecs jouissaient d'une grande popularité dans la région du royaume du Bosphore puisqu'ils constituent largement plus de $90 \%$ des noms transmis par les sources épigraphiques.

Il y a un autre argument qui plaide contre une simple présence des cultures les unes à côté des autres dans cette région. Alors que les vecteurs d'image dans les zones cultuelles - les inscriptions votives - et les lieux de culte eux-mêmes ne présentent guère que des influences grecques, et devraient donc dans un premier temps, théoriquement, être attribués à la population grecque, le rite funéraire des kourganes reflète également une élite sociale qui, s'il fallait la rattacher à un groupe ethnique, devrait être considéré comme non grecque.

$\mathrm{Si}$ on veut attribuer ces kourganes à un groupe ethnique, on devrait les considérer comme non grecs. Or, à l'intérieur des cités, à l'exception des monticules de cendre, on n'a pas, jusqu'à présent, d'indices clairs de l'existence d'une population non grecque: ni l'architecture contemporaine des kourganes ni le petit mobilier n'attestent sa présence. En outre, elle n'utilise pas, semble-t-il, les sanctuaires au centre des grandes villes. Dans celles-ci les monticules de cendre, à en juger par leur taille et leur position, n'ont toutefois jamais joué un rôle central. Si l'on fait le constat d'une juxtaposition des communautés ethniques, d'autres questions se posent: où vivaient donc les bâtisseurs des kourganes et pourquoi ont-ils érigé leurs splendides tombeaux justement le long des voies de communication à l'extérieur des cités, alors que leur présence n'est pas même attestée dans celles-ci? Les prétendus «Grecs» qui habitaient

Labrisa» (qui fournit la bibliographie plus ancienne).

95. CIRB 1137.

96. CIRB 1137: Table A, colonne 1, ligne 5.

97. CIRB 1137: Table B, colonne 1, ligne 24.

98. CIRB 1137: Table A, colonne 1, ligne 52. 
là, en revanche, se mettaient volontiers en scène dans les centres urbains ou les sanctuaires, mais, dans leurs rites funéraires, ils renonçaient apparemment à toute démonstration de prestige. Il nous manquerait donc une élite sociale grecque dans les tombeaux et une élite non grecque dans les villes, y compris les sanctuaires. Une telle reconstruction n'est pas raisonnable. Il faut plutôt imaginer que les usagers des sanctuaires et des monticules de cendre comme les individus enterrés dans les tombes ou reposant dans les kourganes vivaient ensemble dans les grands centres urbains, conçus principalement selon le modèle grec - dans l'état actuel de nos connaissances -, et qu'il s'agissait en partie des mêmes gens. Une séparation des ethnies, irréalisable en pratique, aboutit à des modèles sociologiques inopérants.

Un autre constat s'impose: dans la culture du Bosphore, l'utilisation de tels ou tels éléments culturels n'est pas corrélée à l'appartenance à telle ou telle ethnie. En particulier pour ce qui est de la représentation de soimême, on disposait, dans le domaine cultuel, aussi bien de modèles grecs que non grec, que l'on ne mélangeait guère, semble-t-il, dans la pratique. Sur le territoire du royaume du Bosphore, on recourait aux donations votives, pratique typiquement grecque, pour les divinités grecques, sauf rares exceptions. Les cultes que l'on peut classer comme grecs étaient visiblement menés à la grecque, comme l'indiquent les sources, alors que les cultes des monticules de cendre, dans leur idée et leur structure, ont plus ou moins conservé leur physionomie d'origine, non grecque. Cela ne veut pas dire pour autant que seuls des Grecs s'adonnaient à des pratiques rituelles dans les sanctuaires, et seuls des non-Grecs sur les monticules de cendre. De même, dans les kourganes, n'étaient sûrement pas enterrés seulement des non-Grecs. Ces phénomènes religieux représentent précisément le respect de traditions auxquelles les uns et les autres se sentaient le plus attaché. Toutefois au IV ${ }^{\mathrm{e}}$ s. av. J.-C., on était apparemment plus enclin à utiliser des modèles non grecs sur la péninsule de Taman qu'à Panticapée. Dans la capitale bosporane, on ne peut pas établir de mélange des éléments culturels, que ce soit dans les sanctuaires ou dans les usages cultuels, avant le $\mathrm{II}^{\mathrm{e}}$ s. av. J.-C. C'est alors seulement qu'apparaissent des noms de divinités non grecques dans les consécrations.

Cette perspective permet aussi d'expliquer l'évolution de la communication religieuse et sociale au $\mathrm{IV}^{\mathrm{e}}$ s. av. J.-C., évoquée plus haut, qui se manifeste par l'apparition, semble-t-il, soudaine des inscriptions votives. Si ces inscriptions devaient être attribuées à une seule ethnie, 
elles ne pourraient être le fait que des Grecs, seuls capables de les lire, et ne seraient attestées qu'à partir du IV ${ }^{\mathrm{e}}$ s. av. J.-C., lors de la période de prospérité économique.

Cette phase de prospérité économique du royaume du Bosphore a été marquée par les contacts commerciaux avec Athènes, et par une forte influence des traditions attiques: c'est alors que les charges attiques - telle celle d'agonothète - sont introduites, que les rois portent le titre d'archonte et que, dans plusieurs cités, apparaissent des gymnases et des théâtres comme lieux de culture grecque. Deux autres phénomènes, le recours à la représentation de soi au moyen d'inscriptions votives et la popularité des noms grecs, résultent aussi probablement de cette influence. Les inscriptions votives ne sont donc pas tant le témoignage et le résultat de la puissance financière des dédicants: elles symbolisent plutôt l'adoption de la culture grecque attique sur le territoire du royaume du Bosphore. À partir du III e s. av. J.-C., lorsque sont fondés les sanctuaires tels que les temenos des établissements périurbains de General'skoe Vostočnoe et de la baie toute proche de Sirenevaja et que les inscriptions votives sont en net recul, l'influence attique dans le culte est déjà devenue négligeable. À cette époque, les souverains du Bosphore portent en outre exclusivement le titre de roi et se détournent ainsi nettement des usages précédents, très marqués par la Grèce.

$A u I^{e}$ s. av. J.-C., les habitants du royaume du Bosphore Cimmérien appréhendaient les éléments caractéristiques des différentes cultures présentes sur le territoire de manière réfléchie, en fonction de leur identification avec des représentations religieuses et des attitudes, indépendamment de leur origine ethnique. Les traditions grecques et autres étaient bien distinctes, non seulement dans le domaine religieux, et on recourait aux unes ou aux autres avec assurance et en connaissance de cause. Il s'est ainsi constitué une culture tout à fait particulière, dans laquelle les marques de piété pouvaient se faire aussi bien de manière individuelle, à la grecque, qu'à la manière du "Pont septentrional», dans le cadre d'une communauté cultuelle.

Ivonne OHLERICH Museum am Strom, Bingen 


\section{BIBLIOGRAPHIE}

Aкımova, Ljudmila Ivanovna, "Novyj pamjatnik skul'ptury iz Pantikapeja. K probleme gekatejonov» ["Une nouvelle sculpture de Panticapée. La problématique des Hékatéia»], Vestnik drevnej istorii, 3 (1983), p. 66-87.

Anokhin, Vladilen Afanas'evič, Monetnoe delo Bospora [Le monnayage bosporan], Kiev, Naukova Dumka, 1986.

Artamonov, Mikhail Illarionovič, "K voprosu o proiskhoždenii bosporskikh Spartokidov» "Sur l'origine des Spartocides du Bosphore»], Vestnik drevnej istorii, 1 (1949), p. 29-39.

Astašova, Natalja Sergeevna, et alii, "Drevnejšij Pantikapej Predvaritel'nye itogi arkheologičeskikh issledovanij » [ La Panticapée archaïque - résultats préliminaires des investigations archéologiques»], in Ėlita Bospora i Bosporskaja èlitarnaja kul'tura. Materialy meždunarodnogo kruglogo stola [Élites du Bosphore et culture aristocratique bosporane. Communications de la table ronde internationale], otv. red. Vadim Jur'evič Zuev, Vladimir Andreevič Khršanovskij, Sankt-Peterburg, Palazzo, 2016, p. 92-99.

Belova, Natal'ja Sergeevna, "Novaja nadpis' iz Germonassy" ["Nouvelle inscription de Hermonassa»], Vestnik drevnej istorii, 1 (1967), p. 60-68.

—, «Posvjatitel'naja nadpis' iz Kep» [«Inscription votive de Kèpoi »], Vestnik drevnej istorii, 2 (1970), p. 65-72.

Bessonova, Svetlana Sergeevna, "Kul't Tabiti-Gestii u Skifov» ["Le culte de Tabita-Hestia chez les Scythes»], in Otkritija molodikh arkheologov Ukrainy 1, otv. red. Vladimir Danilovič Baran, Kiev, AN URSS, 1976, p. 24-25.

Blavatskaja, Tat'jana Vasil'evna, "Posvjaščenie Levkona I» ["La consécration de Leukôn I ${ }^{\mathrm{er}}$ », Rossijskaja arkheologija, 2 (1993), p. 34-47.

Blavatskij, Vladimir Dmitrievič, "Materialy po istorii Pantikapeja. Plan goroda» ["Documents servant à l'histoire de Panticapée. Le 
plan de la ville»], Materialy $i$ issledovanija po arkheologii SSSR, 19 (1951), p. 9-62.

—, «O proiskhoždenii bosporskikh sklepov s ustupčatymi perekrytijami » " "De l'origine des tombeaux à voûte à encorbellement dans le Bosphore»], Sovetskaja arkheologija, 24 (1955), p. 29-53.

—, "Ob ètničeskom sostave Pantikapeja v IV-III vv. do n. è." [ «La composition ethnique de Panticapée des IV e et III ${ }^{\mathrm{e}}$ s. av. J.-C.»], Sovetskaja arkheologija, 28 (1958), p. 96-102.

—, Pantikapej. Očerki istorii stolicy Bospora [Panticapée. Essais sur l'histoire de la capitale du Bosphore], Moskva, Nauka, 1967.

Boltunova, Anna Ivanovna, "Novye èpigrafičeskie materialy iz Gorgippii » "Nouveaux documents épigraphiques de Gorgippia»], Vestnik drevnej istorii, 3 (1982), p. 61-66.

Burgunder, Pascal, Semenzato, Camille, "Retour à Alexandrie», in Inscriptions mineures: nouveautés et réflexions. Actes du colloque Ductus (Lausanne 2008), dir. par M. Fuchs et al., Berne, Peter Lang, 2012, p. 283-296.

Butjagin, Aleksandr Mikhajlovič, "Novye issledovanija rannego mirmekijskogo zol'nika» [Nouvelles recherches sur l'autel de cendres de Myrmèkion à époque ancienne»], in Bosporkij Fenomen: Pogrebal'nye pamjatniki i svjatilišca, Materialy meždunarodnoj naučnoj konferencii [Le phénomène bosporan. Monuments funéraires et sanctuaires. Pré-actes de la conférence internationale], otv. red. Vadim Jur'evič Zuev, Sankt-Peterburg, Izdatel'stvo Gosudarstvennogo èrmitaža, 2002, vol. I, p. 90-94.

CIRB = STRUve, Vasilij Vasil'evič, et alii, Korpus bosporskikh nadpisej [Corpus des inscriptions bosporanes], Moskva/Leningrad, Nauka, 1965.

CIRB Al'bom = Gavrilov, Aleksandr Konstantinovič, Korpus Bosporskikh Nadpisej: Al'bom illustracij [Corpus des inscriptions bosporanes: l'album d'illustrations], St-Peterburg, Bibliotheca classica Petropolitana, 2004.

EMEC, Igor' Anatol'evič, Greko-Varvarskie religioznye vzaimovlijanija na Bospore Kimmerijskom [Influences gréco-barbares réciproques dans le Bosphore Cimmérien], Moskva, Kompanija Sputnik, 2002.

Erhardt, Norbert, "Apollon Ietros: Ein verschollener Gott Ioniens?", Istanbuler Mitteilungen, 39 (1989), p. 115-122. 
Frolova, Nina Andreevna, Die frühe Münzprägung vom Kimmerischen Bosporos: Mitte 6. bis Anfang 4. Jh. v. Chr. Die Münzen der Städte Pantikapaion, Theodosia, Nymphaion und Phanagoria sowie der Sinder, Berlin, Akademie-Verlag, 2004.

Gagen, Ljudmila Petrovna, Gavrilenko, Ljudmila Stepanovna, "The study and restoration of antique fresco from Nymphaeum ", in Common Responsibilities and Tasks of the Restorer, Scientist and Museologist in Storing and Exhibiting the Objects of Art, with Particular Regard to the Problems of Exhibit Installations and ShowCases (Fifth international restorer Seminar 1), ed. by Éri Istvan, Budapest, National Centre of Museums, 1985, p. 159-162.

Gajdukevič, Viktor Francevič, "Nekropoli nekotorykh bosporskikh gorodov" [ "Les nécropoles de quelques cités bosporanes »], Materialy $i$ issledovanija po arkheologii SSSR, 69 (1959), p. 154-238.

- "Raboty na Bospore» ["Travaux dans le Bosphore»], Arkheologicheskie otkritija, (1965), p. 100-102.

—, Das Bosporanische Reich, Berlin/Amsterdam, Akademie-Verlag/Adolf M. Hakkert, 1971.

Gerc, Karl Karlovič, Arkheologičeskja topografija Tamanskogo poluostrova [Topographie archéologique de la presqu'île de Taman], Moskva, Izdanie Moskovskago obščestva, 1870.

Grač, Nonna Leonidovna, "Raskopki Nimfeja i ego nekropolja» ["Fouilles de Nymphaion et de sa nécropole»], Arkheologičeskie otkritija 1978 goda, (1979), p. 321-322.

—, «K kharakteristike ètničeskogo sostava naselenija Nimfeja v VI-V vv. do n. è." ["Caractéristiques ethniques de la population de Nymphaion aux $\mathrm{VI}^{\mathrm{e}}-\mathrm{V}^{\mathrm{e}}$ s. av. J.-C.»], in Demografičeskaja situacija v Pričernomor'e v period velikoj grečeskoj kolonizacii. Materialy II Vsesojuznogo Simpoziuma po drevnej istorii Pričernomor'ja [Situation démographique du bassin pontique à l'époque de la colonisation grecque. Pré-actes du deuxième symposium soviétique d'histoire ancienne de la mer Noire], otv. red. Otar Lordkipanidze, Tbilisi, 1981, p. 260-267.

—, «Raskopki Nimfeja» ["Fouilles de Nymphaion»], Arkheologičeskie otkritija 1982 goda, (1983), p. 253-254.

—, «Nimfej v konce IV-I vv. do n. è.» [ "Nymphaion de la fin du IV ${ }^{\mathrm{e}}$ au $\mathrm{I}^{\mathrm{er}}$ s. av. J.-C.»], in Pričernomor'e vèpokhu èllinizma. Materialy III 
Vsesojuznogo Simpoziuma po drevnej istorii Pričernomor' ja [Le bassin pontique à l'époque hellénistique. Pré-actes du troisième symposium soviétique d'histoire ancienne de la mer Noire], otv. red. Otar Lordkipanidze, Tbilisi, Mecniereba, 1985, p. 333-341.

Hintzen-Bohlen, Brigitte, Herrscherrepräsentation im Hellenismus: Untersuchungen zu Weihgeschenken, Stiftungen und Ehrenmonumenten in den mutterländischen Heiligtümern Delphi, Olympia, Delos und Dodona, Köln, Böhlau, 1992.

Höckmann, Olaf, "Naval and other graffiti from Nymphaion", Ancient Civilisation from Scythia to Siberia, 5 (1998), p. 303-356.

Ivanova, Anna Pavlovna, Skul'ptura i živopis' Bospora. Očerki [Sculpture et peinture du Bosphore. Essais], Kiev, Izdatel'stvo Akademii Nauk Ukr. SSR, 1961.

Iwanowa, Anna Pavlovna, "Gestalten der örtlichen Mythologie in der Kunst des Bosporos", Das Altertum, 5 (1959), p. 233-241.

Jajlenko, Valerij Petrovič, "Votiv Levkona I iz Labrisa» ["L’inscription votive de Leukôn I ${ }^{\text {er }}$ à Labrys»], Drevnosti Bospora, 7 (2004), p. 425-445.

Jakovenko, Eleonora Viktorovna, "Kurgan Temir-Gora» ["Le kourgane de Temir-Gora»], Sovetskaja arkheologija, 3 (1972), p. 260-265.

Kastanajan, Elizaveta Grigor'evna, "Gruntovye nekropoli bosporskikh gorodov i mestnye ikh osobennosti" [Les nécropoles à tombes en pleine terre des cités bosporanes et leurs spécificités locales »], Materialy i issledovanija po arkheologii SSSR, 69 (1959), p. 257-295.

Kharko, Lev Petrovič, "Kul't Afrodity na Bospore Kimmerijskom» ["Le culte d'Aphrodite dans le Bosphore Cimmérien»], Kratkie soobšenija o dokladakh $i$ polevykh issledovanijakh Instituta Istorii Material'noj Istorii, 13 (1947), p. 137-141.

Khudjak, Mark Matveevič, "Predvaritel'nye itogi raskopok poslednikh let v Nimfee" "Résultats préliminaires des fouilles de Nymphaion ces dernières années "], in Arkheologija $i$ istorija Bospora [Archéologie et histoire du Bosphore Cimmérien], I, otv. red. Viktor Francevič Gajdukevič, Moskva, Krymizdat, 1952, p. 75-87. —, Iz istorii Nimfeja VI-III vekov do n. è. [De l'histoire de Nymphaion], Leningrad, Izdatel'stvo Gosudarstvennogo èrmitaža, 1962. 
Kocybala, Arcadia Xenia, Greek Colonization on the North Shore of the Black Sea in the Archaic Period, Ann Arbor, University Microfilms International, 1982.

KöHne, Bernhard von, «Über die Marmorbasis aus der Zeit des bosporanischen Königs Pairisades I", Sitzungsberichte der ph.-h. Classe der Königlichen Akademie der Wissenschaften, 11 (1953), p. 399.

Košelenko, Gennadij Andreevič, Kuznecov, Vladimir Dmitrievič, "Grečeskaja kolonizacija Bospora» ["La colonisation grecque du Bosphore»], in Pričernomor'e VII-Vvv. do n. è. Pis'mennye istočniki $i$ arkheologija. Materialy V Meždunarodnogo simpoziuma po drevnej istorii pričernomor' ja [Le bassin pontique du VII au Ve s. av. J.-C. Sources écrites et archéologie. Pré-actes du cinquième symposium soviétique d'histoire ancienne de la mer Noire], otv. red. Otar Lordkipanidze, Tbilisi, Mecniereba, 1985, p. 30-47.

—, "Grečeskaja kolonizacija Bospora» [ "La colonisation grecque du Bosphore»], in Očerki arkheologii $i$ istorii Bospora [Essais sur l'archéologie et l'histoire du Bosphore], otv. red. Gennadij Andreevič Košelenko, Moskva, Nauka, 1992, p. 7-28.

-, "Greek colonisation of the Bosporus", in The Greek colonization of the Black Sea area. Historical interpretation of archaeology. Historia Einzelschriften 121, ed. by Gocha R. Tsetskhladze, Stuttgart, Steiner, 1998, p. 249-262.

KuZ’mina, Julija Nikolaevna, «Organizacija ritualov i žertvoprinošenij v èllinskom svjatilišče» ["Lorganisation des rituels et des sacrifices dans le sanctuaire hellénistique»], Samarskij naučnij vestnik, 4/5 (2013), p. 96-101.

—, «Sakral'nye kompleksy Fanagorii VI-I vv. do n. è.: problemy klassifikacii » " Complexes sacrés de Phanagorie du VI ${ }^{\mathrm{e}}$ au I ${ }^{\mathrm{er}}$ s. av. J.-C.: problèmes de classification»], Problemy istorii, filologii, kul'tury, 3 (2015), p. 208-210.

LJAPUŠKin, Ivan Ivanovič, «Dneprovskoe lesostepnoe levobereže v èpokhu železa» [La rive gauche steppique arborée du Dniepr à l'âge du Fer »], Materialy i issledovanija po arkheologii SSSR, 104 (1961), p. 99-109.

LöHr, Christoph, Griechische Familienweihungen: Untersuchungen einer Repräsentationsform von ihren Anfängen bis zum Ende des 4. Jh. v. Chr., Leidorf, Rahden, 2000. 
Marčenko, Irina Dmitrievna, "K voprosu o kul'takh Aziatskogo Bospora» "À propos des cultes du Bosphore asiatique»], Vestnik drevnej istorii, 2 (1960), p. 101-107.

—, «Novye dannye o dogrečeskom Pantikapee» [ Nouvelles données concernant la Panticapée préhellénique»], Materialy i issledovanija po arkheologii SSSR, 103 (1962), p. 86-93.

—, «Raskopki zdanija II v. do. n. è. v Pantikapee 1963-1965» [«Fouilles du bâtiment du II e s. av. J.-C. à Panticapée en 1963-1965»], Kratkie soobščenija, 109 (1967), p. 79-87.

—, "O kul'te Afrodity na Tamane» ["Le culte d'Aphrodite sur la péninsule de Taman»], in Istorija i kul'tura antičnogo mira [Histoire et culture de monde antique], otv. red. Ekaterina Mikhajlovna Alekseeva, Moskva, Nauka, 1977, p. 121-126.

MarČEnKo, Konstantin Konstantinovič, "Osnovnye aspekty i resul'taty izučenija greko-varvarskikh kontaktov i vzaimodejstvij v Severnom Pričernomor'e skifskoj èpokhi» ["Aspects principaux et résultats de l'étude des contacts et interactions gréco-barbares dans le Pont Nord à l'époque scythe»], Skifskij kvadrat, 3 (1999), p. 333-354.

Maslennikov, Aleksandr Aleksandrovič, Naselenie Bosporskogo Gosudarstva v VI-II vv. do. n. è. [La population de l'État bosporan du VIe au II s. av. J.-C.], Moskva, Nauka, 1981.

—, Ellinskaja khora na kraju Ojkumeny [Une chôra hellénistique à la périphérie de l'oikouménè], Moskva, Izdatel'stvo Indrik, 1998.

—, Sel'skie svjatilišča evropejskogo Bospora [Les sanctuaires ruraux du Bosphore européen], Tula, Grifon i K, 2007.

Molev, Evgenij Aleksandrovič, Bospor v period èllinizma [Le Bosphore à l'époque hellénistique], Nižnij Novgorod, Izdatel'stvo Nižegorodskogo Universiteta, 1994.

Moleva, Natal'ja Vladimirovna, "Kitejskoe svjatilišče» [ "Le sanctuaire de Kytaia»], Ritual i ritual'nij predmet, Gosudarstvennyj muzej istorii religii i ateizma [Rituel et objet du rituel. Musée d'histoire des religions et de l'athéisme], (1995), p. 42-49.

—, Očerki sakral'noj žizni Bospora [Essai sur la vie religieuse du Bosphore], Nižnij Novgorod, 2002.

Morrison, John S., Greek and Roman oared worships, Oxford, Oxbow Books, 1996. 
Noonan, Thomas S., "The origins of the Greek colony at Pantikapaeum ", American Journal of Archaeology, 77 (1973), p. 77-81.

Ohlerich, Ivonne, Kultorte und Heiligtümer auf dem Gebiet des Bosporanischen Reiches. Vom Beginn der Kolonisation bis zum Ende des 2. Jh. v. Chr., thèse de doctorat, Rostock, 2009; le manuscrit est disponible via <http://rosdok.uni-rostock.de/resolve/id/ rosdok_disshab_0000000605> [consulté le 27 août 2019].

Paromov, Jakov Maksimovič, "Kurgannyj nekropol' Germonassy» [«La nécropole tumulaire de Hermonassa»], Drevnosti Bospora, 4 (2002), p. 192-206.

Podossinov, Alexander Vasil'evič, "Am Rande der griechischen Oikumene. Geschichte des Bosporanischen Reiches", in Das Bosporanische Reich. Der Nordosten der Schwarzen Meeres in der Antike, hrsg. von Jochen Fornasier, Burkhard Böttger, Mainz, Philipp von Zabern, 2002, p. 21-38.

Rostovcev, Mikhail Ivanovič, «Iranskij konnyj bog i Jug Rossii » [ Le dieu équestre iranien et la Russie méridionale»], Vestnik drevnej istorii, 2 (1990), p. 192-196.

Rostovtzeff, Mikhail, "Le culte de la Grande Déesse dans la Russie Méridionale", Revue des études grecques, 32 (1921), p. 462-481.

-, Iranians and Greeks in South Russia, Oxford, Clarendon Press, 1922. Rozanova, Nina Pavlovna, «Posvjatitel'naja nadpis' Sanergu i Astarte. Pamjatnik caricy Komosarii " " Une inscription votive à Sanergès et Astarté. Le monument de la reine Komosarya »], Vestnik drevnej istorii, 2 (1949), p. 86-93.

—, «Posvjatitel'nye nadpisi Fanagorii kak istočnik dlja topografii goroda" [Les inscriptions votives de Phanagorie comme source pour la topographie de la ville»], Vestnik drevnej istorii, 3 (1949), p. 171-178.

—, «K voprosu o mestonakhoždenii Apatur» ["À propos de la localisation d'Apatouron»], Vestnik drevnej istorii, 2 (1951), p. 210-213.

Rusjaeva, Anna Stanislavovna, "Milet-Didimy-Borisfen-Ol'vija. Problemy kolonizacii nižnego pobuž ja» ["Milet - Didyme Borysthène - Olbia. Colonisation de la région du Bug inférieur »], Vestnik drevnej istorii, 2 (1986), p. 25-64.

—, «Feb Apollon na Bospore» [Phébus Apollon dans le Bosphore»], in Bospor Kimmerijskij i varvarskij mir v period antičnosti $i$ 
srednevekov' ja [Le Bosphore Cimmérien et le monde barbare dans l'Antiquité et au Moyen Âge], otv. red. Viktor Nikolaevič Zin'ko, Kertch, Kerčenskaja Gorodskaja Tipografija, 2003, p. 225-229.

ŠAUB, Igor' Jurevič, Kul'ty i religioznye predstavlenija naselenija Bospora VI-IV vekov do n. è. [Les cultes et les représentations religieuses de la population bosporane du VIe au IV e s. av. J.-C.], Sankt-Peterburg, Akademija Istoričeskikh Nauk, 1987.

—, "Kul't velikoj bogini u mestnogo naselenija Severnogo Pričernomor’ ja» [ "Le culte de la grande déesse auprès de la population indigène du Pont septentrional»], Stratum Plus, 3 (1999), p. 207-223.

ŠčEgLov, Aleksandr Nikolaevič, "O greko-varvarskikh vzaimodejstvijakh na periferii èllinističeskogo mira» [«Interactions grécobarbares à la périphérie du monde grec»], in Pričernomor'e $v$ èpokhu èllinizma. Materialy III Vsesojuznogo Simpoziuma po drevnej istorii Pričernomor' ja [Le bassin pontique à l'époque hellénistique. Pré-actes du troisième symposium soviétique d'histoire ancienne de la mer Noire], otv. red. Otar Lordkipanidze, Tbilisi, Mecniereba, 1985, p. 185-198.

Schlotzhauer, Udo, Žuravlev, Denis V., «Forschungen in der antiken Siedlung ,, Golubickaja-2 “ auf der südrussischen Taman'Halbinsel ", Eurasia Antiqua, 19 (2013), p. 105-125.

Schmidt-Dounas, Barbara, Geschenke erhalten die Freundschaft: Politik und Selbstdarstellung im Spiegel der Monumente, Berlin, AkademieVerl., 2000.

ŠELov, Dmitrij Borisovič, «K voprosu o vzaimodejstvii grečeskikh i mestnykh kul'tov v Severnom Pričernomor'e» ["Influences réciproques des cultes grecs et locaux dans le Pont septentrional»], Kratkie Soobšcenija o dokladakh i polevykh issledovanijakh Instituta Istorii Materil'noj Kul'tury [Communications brèves sur les exposés et les instigations de terrain de l'Institut d'histoire de la culture matérielle], 34 (1950), p. 62-69.

—, "Istorija" ["Histoire»], in Antičnye gosudarstva Severnogo Pričernomor' ja [Les États antiques du Pont septentrional], otv. red. Gennadij Andreevič Košelenko et alii, Moskva, Nauka, 1984, p. 8-22.

Šelov-Kovedjaev, Fjodor Vadimovič, «Proščanie s Ditagojej. Konec odnogo i roždenija novogo božestva» ["L'adieu à Ditagoia. La fin 
d'une divinité et la naissance d'une nouvelle»], Aristej, 16 (2017), p. 107-115.

Silant'eva, Lina Fjodorovna, «Nekropol’ Nimfeja» [«La nécropole de Nymphaion"], Materialy i issledovanija po arkheologii SSSR, 69 (1959), p. 5-107.

SkržInskaja, Marina Vladimirovna, "Bosporjane na prazdnikakh Apollona» [Les Bosporans aux fêtes d'Apollon»], in Bospor Kimmerijskij i varvarskij mir $v$ period antičnosti i srednevekov' ja [Le Bosphore Cimmérien et le monde barbare dans l'Antiquité et au Moyen Âge], otv. red. Viktor Nikolaevič Zin'ko, Kertch, Kerčenskaja Gorodskaja Tipografija, 2003, p. 230-233.

Sokolova, Ol'ga Jur'evna, «Raboty nimfejskoj èkspedicii» ["Travaux de la mission archéologique de Nymphaion "], in Arkheologičeskie issledovanija v Krymu 1993 god. Sbornik naučnikh statej [Investigations archéologiques en Crimée en 1993. Recueil d'articles scientifiques], otv. red. Vadim Aleksandrovič Kutajsov, Simferopol', Institut Arkheologii - Krymskij filial, 1994, p. 240-241.

-, "Investigations of an early hellenistic shrine in Nymphaeum", Ancient Civilizations from Scythia to Siberia, 6 (1998), p. 155-172.

—, "Nymphaeum", in Ancient Greek colonies in the Black Sea, II, ed. by Demetrios V. Grammenos, Thessaloniki, Archaeological Institute of Northern Greece, 2003, p. 759-802.

—, "Lučšie nakhodki iz raskopok Nimfeja za poslednie desjat' let» ["Les meilleures trouvailles des fouilles de Nymphaion durant la décennie écoulée»], Soobščenija Gosudarstvennogo èrmitaža [Communications du Musée d'État de l'Ermitage], 62 (2004), p. 91-96.

Sokolova, Ol'ga Jur'evna, Pavličenko, Natal'ja Andreevna, «Novaja posvjatitel'naja nadpis' iz Nimfeja» ["Nouvelle inscription votive de Nymphaion »], Hyperboreus, 8.1 (2002), p. 99-121.

Sokol'skiJ, Nikolaj Ivanovič, «Svjatilišče Afrodity v Kepakh» [ "Le sanctuaire d'Aphrodite à Kèpoi »], Sovetskaja Arkheologija, 4 (1964), p. 101-118.

-, Tamanskij Tolos i residencija Khrisaliska [La tholos de Taman et la résidence de Chrysalisque], Moskva, Nauka, 1976.

StÄHler, Klaus, "Die antike Siedlung Vyšesteblievskaja 1 ", in Das Bosporanische Reich. Der Nordosten der Schwarzen Meeres in der 
Antike, hrg. von Jochen Fornasier, Burkhard Böttger, Mainz, Philipp von Zabern, 2002, p. 113-120.

Surikov, Igor' Evgen'evič, "Historico-geographical questions connected with Pericle's pontic expedition ", Ancient Civilizations from Scythia to Siberia, 7 (2001), p. 341-366.

Tokntas'ev, Sergej Remirovič, "Bosporskaja legenda ob Afrodite Apaturos» ["Légende bosporane sur Aphrodite d'Apatouron »], Vestnik drevnej istorii, 2 (1983), p. 111-117.

—, "Apatur. Istorija Bosporskogo svjatiliščja Afrodity Uranii» [«Apatouron. Histoire du sanctuaire bosporan à Aphrodite Ourania»], Vestnik drevnej istorii, 2 (1986), p. 138-145.

Tolstikov, Vladimir Petrovič, «K probleme obrazovanija Bosporskogo gosudarstva» ["À propos de la formation de l'État bosporan»], Vestnik drevnej istorii, 3 (1984), p. 24-48.

-, "Pantikapaeum: The capital of the Kingdom of Cimmerian Bosporus in the light of new archaeological studies", in Ancient Greek colonies in the Black Sea, II, ed. by Demetrios V. Grammenos, Thessaloniki, Archaeological Institute of Northern Greece, 2003, p. 707-758.

—, «Khram Apollona na akropole Pantikapeja: Problemy datirovki, tipologii i periodizacii» [«Le temple d'Apollon sur l'acropole de Panticapée: problèmes de datation, de typologie et de périodisation »], Problemy istorii, filologii, kultury, 1 (2010), p. 277-315.

Tolstikov, Vladimir Petrovič, Kuz’mina, Julija Nikolaevna, «Ein neuer Baubefund des 4.-3. Jh. v. Chr. im Zentrum von Pantikapaion", Eurasia Antiqua, 17 (2011), p. 215-232.

Tolstikov, Vladimir Petrovič, Lomtadze, Georgij Al’bertovič, «K voprosu o vremeni osnovanija basilej Spartokidov na akropole Pantikapeja" ["Chronologie de la construction du palais des Spartocides sur l'acropole de Panticapée»], Drevnosti Bospora, 4 (2001), p. 427-441.

Ustinova, Yulia, "Aphrodite Ourania of the Bosporus: The great goddess of a frontier pantheon", Kernos, 11 (1998), p. 209-226.

-, The supreme gods of the bosporan kingdom: Celestial Aphrodite and the most high god, Leiden, Brill, 1999.

—, "Apollo Iatros: A greek god of pontic origin", in Die Griechen und ihre Nachbarn am Nordrand des Schwarzen Meeres. Beiträge des 
Internationalen archäologischen Kolloquiums 2001, hrsg. von Klaus Stähler, Gabriele Gudrian, Münster, Ugarit, 2009, p. 246-298.

Vinogradov, Jurij Germanovič, "Die historische Entwicklung der Poleis des nördlichen Schwarzmeergebietes im 5. Jahrhundert v. Chr.», Chiron, 10 (1980), p. 64-100.

—, «Votivnaja nadpis’ dočeri carja Skilura iz Pantikapeja i problemy istorii Skifii i Bospora vo II v. do n. è.» [«Une inscription votive de la fille du roi Skilouros découverte à Panticapée et la problématique de l'histoire de la Scythie et du Bosphore au $\mathrm{II}^{\mathrm{e}} \mathrm{s}$. av. J.-C.»], Vestnik drevnej istorii, 1 (1987), p. 55-87.

Waldhauer, Oskar, Die Antiken Skulpturen der Ermitage, III, Berlin, de Gruyter, 1936.

WĄsow ICZ, Aleksandra, "Nymphaion: Kolonia grecka na Krymie» ["Nymphaion: une colonie grecque en Crimée»], Meander: Miesięcznik poświęcony kulturze świata starożytnego, 49 (1994), p. 155-166.

Zavojkin, Aleksej Andreevič, "Pamjatnik Satira I na Aziatskom Bospore» [ "Un monument de Satyros I ${ }^{\text {er }}$ dans le Bosphore asiatique»], Drevnosti Bospora, 3 (2000), p. 47-62.

Zgusta, Ladislav, Die Personennamen griechischer Städte der nördlichen Schwarzmeerküste. Die ethnischen Verhältnisse, namentlich das Verhältnis der Skythen und Sarmaten im Lichte der Namensforschung, Praha, Nakladatelstvi Československé Akademie věd, 1955.

—, "Die verschiedenen Bevölkerungsschichten der griechischen Städte des nördlichen Schwarzmeergebietes", in Griechische Städte und einheimische Völker des Schwarzmeergebietes: eine Aufsatzsammlung, hrsg. von Johannes Irmscher, Dimitrij B. Schelow, Berlin, Akad.-Verl., 1961, p. 158-163.

ZıN’ко, Viktor Nikolaevič, "Bosporkij gorod Nimfej i varvary» [«La cité bosporane de Nymphaion et les Barbares»], Bosporkie Issledovanija, 1 (2001), p. 207-214.

—, «Khora Nimfeja v VI-IV vv. do n. è.» [ «La chôra de Nymphaion du $\mathrm{VI}^{\mathrm{e}}$ au IV ${ }^{\mathrm{e}}$ s. av. J.-C.»], Drevnosti Bospora, 1 (1998), p. 86-89. 
